\title{
Encoding of spatial information in images of an outdoor scene by pigeons and humans
}

\author{
MARCLA L. SPETCH, DEBBIE M. KELLY, and DAVID P. LECHELT \\ University of Alberta, Edmonton, Alberta, Canada
}

\begin{abstract}
Pigeons and adult humans searched for a 2-cm ${ }^{2}$ unmarked goal in digitized images of an outdoor scene presented on a touch-screen monitor. In Experiment 1, the scene contained three landmarks near the goal and a visually rich background. Six training images presented the scene from different viewing directions and distances. Subsequent unreinforced tests in which landmark or background cues were removed or shifted revealed that pigeons' search was controlled by both proximal landmarks and background cues, whereas humans relied only on the proximal landmarks. Pigeons' search accuracy dropped substantially when they were presented with novel views of the same scene, whereas humans showed perfect transfer to novel views. In Experiment 2, pigeons with previous outdoor experience and humans were trained with 28 views of an outdoor scene. Both pigeons and humans transferred well to novel views of the scene. This positive transfer suggests that, under some conditions, pigeons, like humans, may encode the three-dimensional spatial information in images of a scene.
\end{abstract}

Many organisms have been shown to use visual landmarks to remember and locate a goal area (see Collett, 1992, and Gallistel, 1990, for reviews). Use of visual landmarks is demonstrated experimentally by manipulating the visual cues between the opportunity to encode the spatial information and subsequent search tests in which the subject attempts to locate the goal. Two types of evidence indicate that a subject uses a particular landmark: (1) the subject shifts its search location in response to landmark shifts, and (2) accurate search is disrupted by the absence of the landmark. In a series of experiments using a laboratory task in which food is hidden on the floor of a spatial arena, Cheng and his colleagues (e.g., Cheng, 1988, 1989, 1994; Cheng \& Sherry, 1992) have clearly demonstrated that pigeons use visual landmarks to locate a hidden goal, and they have identified several principles of pigeons' landmark-based search.

Pigeons' landmark-based search has also been investigated in a touch-screen task in which pigeons search for an unmarked goal on the surface of a monitor (Spetch, Cheng, \& Mondloch, 1992; Spetch \& Mondloch, 1993; Spetch \& Wilkie, 1994). Visual stimuli displayed on the monitor served as landmarks. This two-dimensional (2-D)

This research was supported by a Natural Sciences and Engineering Research Council of Canada research grant held by the first author. Some of the data from Experiment 1 were presented at the Conference on Comparative Cognition, Melbourne, FL, in March 1993, and at the meeting of the Psychonomic Society, Los Angeles, CA, in November 1995. The results of Experiment 2 were presented at the International Conference on Comparative Cognition, Melbourne, FL, in March 1997. The authors thank R. Kelly and A. Soldat for assistance with the research and D. Treit for comments on an earlier version of the manuscript. Correspondence should be addressed to M. L. Spetch, Department of Psychology, University of Alberta, Edmonton, AB, Canada T6G 2E9 (e-mail: mspetch@psych. ualberta.ca).

-Accepted by previous editor, Robert A. Rescorla search task has yielded results that are remarkably similar in certain ways to those found in real-space tasks. In particular, near landmarks are weighted more heavily than are far landmarks (Cheng, 1989; Spetch \& Wilkie, 1994), and landmarks near an edge of the search space exert more control in the dimension parallel to the edge than in the dimension perpendicular to the edge (Cheng \& Sherry, 1992; Spetch et al., 1992). Recent work (Spetch, Cheng, \& MacDonald, 1996; Spetch et al., 1997) has revealed that, on both the touch screen and the laboratory floor, pigeons can use the configuration of an array of landmarks to locate a goal. In both environments, however, they respond to expansions of the array by maintaining the absolute training distance from individual landmarks in the array, rather than adjusting distance so as to maintain the appropriate relative position with respect to the entire array of landmarks.

Although the results obtained to date suggest that the touch-screen task calls forth many of the spatial processes used in more naturalistic search tasks, the visual information provided in studies using the touch-screen task has clearly differed from that in most real-world spatial search situations. In particular, whereas landmarks found in natural settings are typically located within a rich visual context consisting of many distal as well as local stimuli, the landmarks presented in the touch-screen studies have been presented against visually uniform backgrounds. In most studies, the landmarks have been colored 2-D geometric stimuli presented against a uniform gray background (Cheng \& Spetch, 1995; Spetch, 1995; Spetch et al., 1992; Spetch \& Mondloch, 1993). One study (Spetch \& Wilkie, 1994) presented visually richer stimuli in the form of digitized images of an outdoor scene consisting of three objects on a grassy field. However, the grassy field was reasonably uniform, except for a gradient in the texture of the grass from the top (background) to the bottom (foreground) of the image. Most importantly, 
there were no objects in the background of the scene that would provide a spatial context for the landmarks.

Distal background cues in a scene could play an important role in spatial search. For example, distal cues may be used to define the general region in which to search, with local landmarks serving to pinpoint the goal within that region. Distal cues may also provide a context that determines the meaning of local landmarks (e.g., the clump of trees beside the river may signify something different than a similar looking clump of trees in an open field). Finally, distal cues may be used as additional redundant sources of information to locate a goal. Indeed, laboratory studies of spatial memory have suggested that both global and local cues may be encoded to remember a goal location (Brodbeck, 1994; Spetch \& Edwards, 1988).

Another way in which the visual information during real-world search is very different from that available in the touch-screen studies is that a goal area can often be approached from different directions in the real world. This means that the visual information used in searching for the goal changes because it is viewed from different perspectives. The touch-screen studies published to date, however, have provided only a single perspective of the landmark array.

The present research used the touch-screen task to investigate pigeons' spatial search in images of outdoor scenes that included landmarks near the goal and a visually rich set of background cues. In Experiment 1, subjects were trained with six images that provided some variation in direction and distance from which the scene was viewed. This meant that the three-dimensional (3-D) spatial relationships in the scene depicted by the images were invariant, but the 2-D spatial relationships among the visual features varied somewhat from image to image. A variety of tests were conducted following training to determine what information in the scene was used when searching for the goal and whether accurate searching would transfer to novel views of the same scene. For comparison, adult humans were trained with the same set of training images and then were given some of the same tests presented to pigeons.

In Experiment 2, racing pigeons with prior outdoor experience were trained with 28 different views of another complex scene and then were tested for transfer to novel views. Again, humans were trained and tested with the same stimuli for comparison.

\section{EXPERIMENT 1}

\section{Method}

\section{Subjects}

Pigeons. Four adult Silver King pigeons served in Experiment 1. One pigeon was experimentally nave, and 1 had previous experience in standard operant chambers but had never served in a touchscreen task. The other 2 pigeons were experienced at performing in the touch-screen spatial search task, but with visual stımuli different from those used here. The birds were housed in large individual cages under a 12-h light:dark cycle (lights on at 6:00 a m.). All birds were maintained at approximately $85 \%$ of their free-feeding weights by Kay Tee pıgeon pellets obtained after experımental sessions and mixed grain durnng experimental sessions. Water and grit were avallable ad lib in the home cages.

Humans. The human subjects were 8 female undergraduate students, ranging from 19 to 47 years of age. They participated in the experiment for credit for their introductory psychology course. Four partıcipants were assigned to the landmark removal test serıes, and 4 were assigned to the transfer test series.

\section{Apparatus, Search Space, and Images}

Pigeons. The experiment was conducted in a large custom-built chamber, $44 \mathrm{~cm}$ high, $32 \mathrm{~cm}$ deep, and $74 \mathrm{~cm}$ wide (inside dimensions). A Zenith 1492 color monitor with attached infrared touch frame (Carroll Touch, 1492 Smart Frame) was placed against an openıng centered in the back wall of the chamber. This opening was $10 \mathrm{~cm}$ from the rassed grid floor of the chamber, and it provided access to the entire surface of the monitor. Spacers were used to recess the touch frame by approximately $3 \mathrm{~cm}$ from the opening and to separate the frame from the monitor by approximately $1.6 \mathrm{~cm}$. Two Gerbrands pigeon grain feeders were mounted on the back wall, one on each side of the monitor. The feeder openings began $8.5 \mathrm{~cm}$ from the sides of the monitor opening and $17 \mathrm{~cm}$ from the floor. Lamps located within each feeder illuminated feeder presentations, and photocells in each hopper measured head entries so as to limit eatıng tımes. Microcomputers, located in an adjacent room, controlled experimental contingencies and recorded peck coordinates. The touch frame was programmed to detect individual responses (i.e., detection of a beam break, then a return to unbroken beams before another response would be recorded).

The search space was a rectangular area, approximately $26 \times$ $20 \mathrm{~cm}$, on the surface of the color monitor The images displayed durıng trainıng provided six different views of an outdoor scene that contained three landmarks (a chair, a pot of flowers, and a pile of logs) near the goal and several visually distinct background cues (house, flowers, trees, etc.). These six views are shown in Figure 1 The rectangular object that can be seen near the three landmarks was a blue plastıc box that served as a marker for the goal area. This goal marker was present in the images only during preliminary training. Thereafter, it was edited out of the images so that it was absent from the scene during the later stages of training and durıng all testıng. Note that the six different views used in training not only provided variation in the vantage point of the scene but also provided variation in the absolute location on the screen of the goal.

The images were digitized and constructed using procedures sımılar to those described in Spetch and Wilkıe (1994) and Wilkıe, Mak, and Saksida (1994). The landmarks and goal marker were la1d out, and the scene was videotaped from various viewpoints. The videotape was then played into a computer using a frame grabber system (Creative Labs Video Blaster), and stıll frames were saved in a GIF format (Compuserve, Inc). Six of the saved images were selected for use in training, and an additional set of six images was selected for use in transfer tests. Removal of the goal marker from the images and manipulation of the visual information in the scenes for the various test phases were accomplished by editing the images with Photofinish software (Zsoft).

For each view, the location of the goal needed to be matched to the touch-screen coordinates. This matching was done by displaying the scene with the goal marker present, touching in the center of the goal marker, and reading off the coordinates recorded by the touch frame This was repeated several times for each image to ensure reliability

Humans. The experıment took place in a small private room that contained a touch-screen-equipped computer. The computer monitor (Zenith 1490) and touch screen (Carroll Touch 1490 Smart Frame) provided the same search space and stimul as those used for the pigeons in Experiment 1. The subjects sat in a chair in front of the monitor and searched by touching the screen with the eraser end of a pencil. 


\section{Training Images}

1

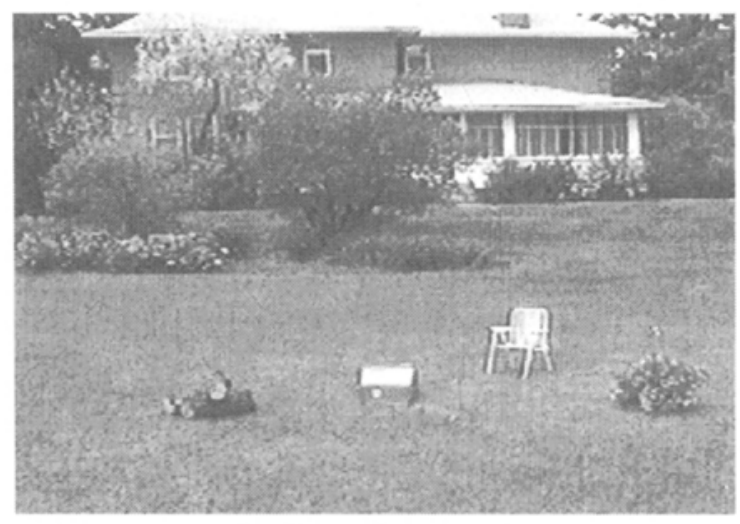

3

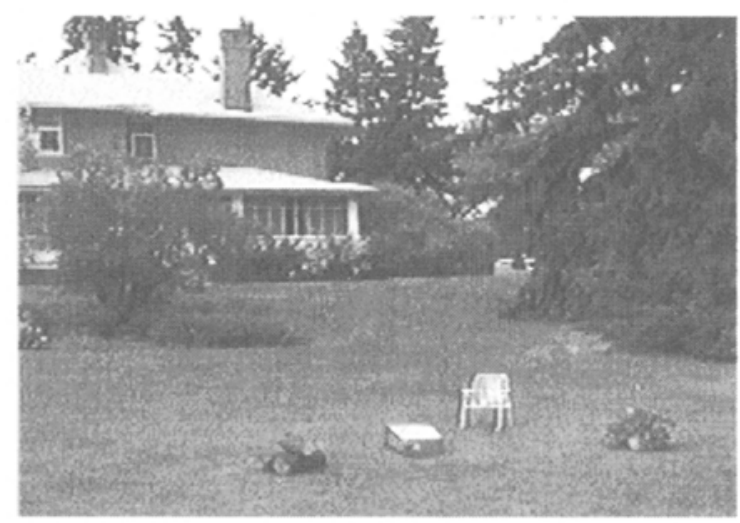

5

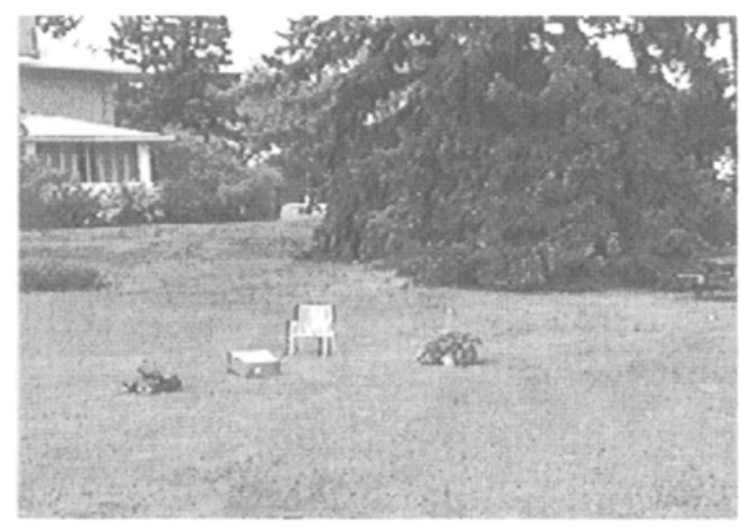

2

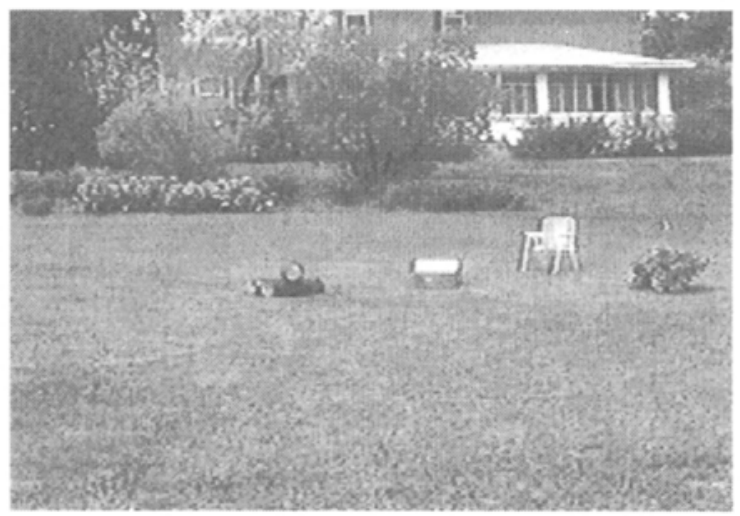

4

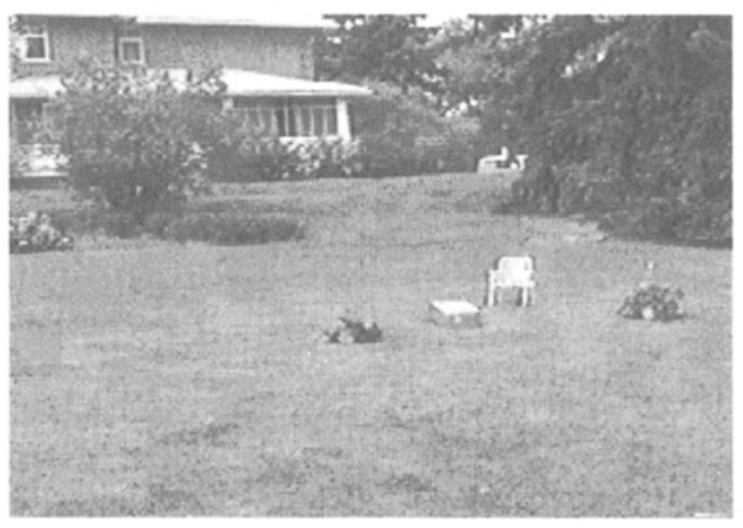

6

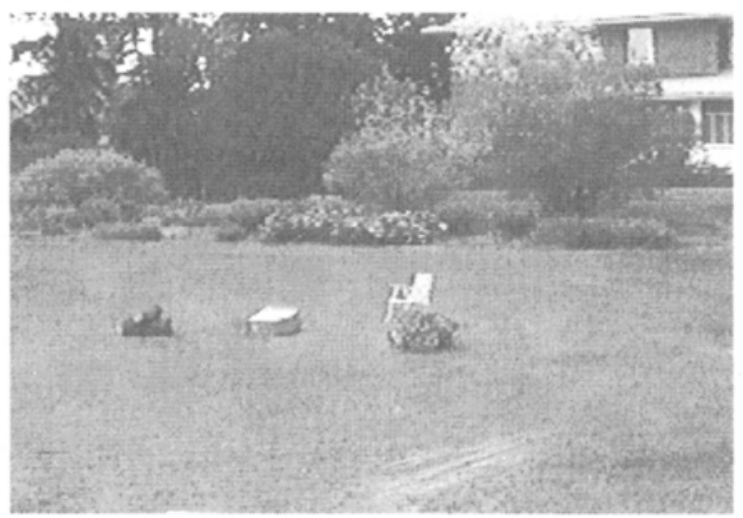

Figure 1. Images showing the six training views used for the subjects in Experiment 1. The goal marker (the box located between the chair and logs) is shown in these images for illustrative purposes. This marker was edited out of the images after preliminary training and was not present during any baseline or test trials.

\section{Procedure for Pigeons}

Preliminary training. The 2 pigeons with no previous experience in the touch-screen task received one or two sessions of training to eat from the raised illuminated hopper. All birds then received several sessions of training with a modified autoshapıng procedure to establish reliable pecking at the monitor Initially, a $2-\mathrm{cm}$ yellow square was intermittently presented in various screen locations against a dark gray background, with 60-sec intertrial intervals (ITIs). The yellow square remained on untıl the bird pecked at the square or untll $8 \mathrm{sec}$ elapsed, and then food was presented. Once reliable peckıng was established to the yellow square, the background was changed to green and the square was changed to blue. Once a bird pecked reliably at the blue square, the six images shown in Figure 1 were introduced. Pecks in a $2-\mathrm{cm}$ area centered at the blue box that 
served as the goal marker were reinforced immediately; otherwise, the display terminated with food after $8 \mathrm{sec}$. For the final session of preliminary training, the ITI was decreased to $5 \mathrm{sec}$, and the image stayed on until a peck in the goal area was recorded.

Search training. During initial search training sessions, the goal marker was gradually eliminated so that the pigeons needed to rely on visual cues in the scene to find the goal. The marker was removed by editing the images to cover the marker with patches of grass using a "clone" tool. Images were created in which the grass covered about $50 \%, 75 \%$, and, finally, $100 \%$ of the marker (the control image in Figure 2 shows an example of an image with the marker $100 \%$ covered). This phase of search training continued until the bird completed (by pecking in the goal area) at least 80 trials in a 60 -min session with the goal marker absent. During the next few sessions, the number of pecks in the goal required to terminate the trial and obtain food was increased to two and then to three. Next, a consecutive peck requirement was added such that the last two pecks recorded had to be consecutive pecks in the goal area. Thus, the response requirement during the final stage of training and for all subsequent baseline sessions was a minimum of three pecks in the goal with the final two pecks being consecutive pecks in the goal. Each increment in the response requirement was implemented only if the bird completed 80 trials in a session. During the last set of search training sessions, the percentage of trials on which reinforcement was available was decreased from $100 \%$ to $50 \%$. On unreinforced trials, completion of the response requirement terminated the display, but no food was presented. Each bird remained on the $50 \%$ reinforcement condition for a minimum of 5 sessions and advanced to the test phase only after it completed at least 80 trials on each of the last two sessions. Search training required between 50 and 70 sessions.

Testing. All birds received several series of test sessions. Two or more baseline sessions, identical to the last phase of training, were inserted between each test series. Baseline sessions were also interspersed among the test sessions (typically, one baseline session after every 3rd or 4th test session) within each test series. Each test session consisted of a mixture of reinforced and unreinforced baseline trials, control trials that were visually identical to baseline trials but were procedurally the same as test trials, and test trials in which visual information in the image was altered in some way. On control and test trials, the image remained on for $8 \mathrm{sec}$ following the second peck recorded anywhere on the screen. In all test sessions, at least $50 \%$ of the trials were reinforced baseline trials. Each type of scheduled test trial occurred once in each block of 15 or 16 trials, with the order varying randomly between blocks.

For one test series, the images were edited to remove one or two of the local landmarks. These tests provided an assessment of accuracy with each landmark removed from the scene and with each landmark being the only local landmark present in the scene. Removal of the landmark was accomplished by cloning grass on top of the landmark. Test and control trials were conducted with each of the six training views.

For a second test series, the images were edited to remove all three local landmarks (see "No Lms" image in Figure 2), to remove all of the background information (see "No Bk" image in Figure 2), or to remove both the landmarks and the background (see "None" image in Figure 2). These removals were also accomplished by copying grass over the landmarks or background. Test and control trials were again conducted with each of the six training views.

For a third test series, the set of three landmarks was shifted hor1zontally in some tests and vertically in others. Only Views 2, 4, 5 , and 6 were used for these tests. Vertical shifts were always down. Horizontal shifts were to the left in Views 2 and 4 and to the right in Views 5 and 6 . All vertical shifts were by $2 \mathrm{~cm}$. The horizontal shifts were by $2 \mathrm{~cm}$ in Views 1,4 , and 5 , but by error were only $1 \mathrm{~cm}$ in View 6. The image labeled "Lm Shift" in Figure 2 shows an example of a leftward shift of the landmarks in View 4. Shifts were accomplished by cloning the area containıng the landmarks to the de- sired location and then clonıng grass over the top of whatever remained of the original landmarks.

For a fourth test series, the backgrounds were swapped for three pairs of training views. This was accomplished by cloning the background of one image (the entire portion of the image above the landmarks) onto the other image. An example of View 4 containing the background of View 1 is shown as the image labeled "Bk Swap" in Figure 2.

The final test series assessed whether the birds would transfer the learned spatial relationships from the set of six training views to a new set of images depicting the same scene but from different distances, perspectives, or orientations. The six images used for the transfer tests are shown in Figure 3. Each test session included $50 \%$ reinforced trials with the trained views, $25 \%$ unreinforced control trials in which each of the trained views occurred equally often, and $25 \%$ unreinforced test trials in which each of the transfer test images was presented equally often. Each bird received four transfer test sessions, each separated by a baseline session.

In all series, each individual test image (i.e., each test type in each view) was presented at least 10 times.

\section{Procedure for Humans}

The images used for the human subjects were the same as those used for the pigeons. At the beginning of each session, the subjects were provided with the following information: Their task would be to remember and locate a goal area, which initially would be marked but later would be unmarked. A point would be available on randomly selected trials. They could obtain this point only by touching in the goal area. The required number of touches in the goal would vary across trials, and sometimes consecutive touches in the goal would be required. Whenever they earned a point, their cumulative points would be displayed. When points were not available, the trial would end after a certain number of touches, whether or not they were correct. The session would end after they obtained a certain number of points or after $45 \mathrm{~min}$, whichever came first.

The experimenter then ran a demonstration program that presented three training trials. The experimenter demonstrated the importance of holding the pencil straight and removing it between touches. The first two demonstration trials presented images with the marker present, and the last demonstration trial presented an image with the marker absent. Two touches in the goal were required during each demonstration trial. Each trial ended as a "reinforced" trial in which a point was earned (i.e., the screen was blanked and cumulative points were displayed). Any procedural questions were answered with "I'm sorry but I cannot provide any further information until after you have completed the experiment." After confirming that the subject wished to participate, the experimenter started the program and left the room.

The subject's program began with 6 trials that presented each of the six training images with the goal marker present. The next 6 trials presented images showing the same views but with the marker absent. This was followed by 6 more trials with the marker present. The marker was absent in all remaining trials of the session. The response requirement for all baseline trials included a variable number of minimum touches (one to five) and a variable number of consecutive touches in the goal (ranging from no consecutive touch requirement to three consecutive touches required). Beginning with Trial 13 , only $50 \%$ of the trials were reinforced (i.e., earned points). On unreinforced baseline trials, the display ended with a blank screen upon completion of the response requirement.

Beginnıng with Trial 31 , control and test trials were interspersed among the baseline trials. During control and test trials, the second touch recorded anywhere on the screen initiated an interval that varied randomly from 2 to $4 \mathrm{sec}$, after which the first response anywhere on the screen terminated the trial. Each of the six training views was presented equally often on control trials and served equally as the basis for test images. The subjects assigned to the removal test series were tested with images in which all three local landmarks 


\section{Test Images}

\section{Control}

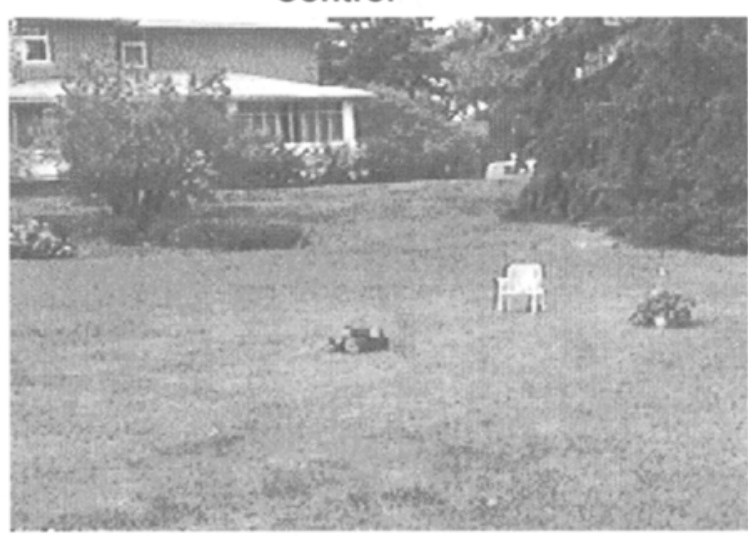

No Bk

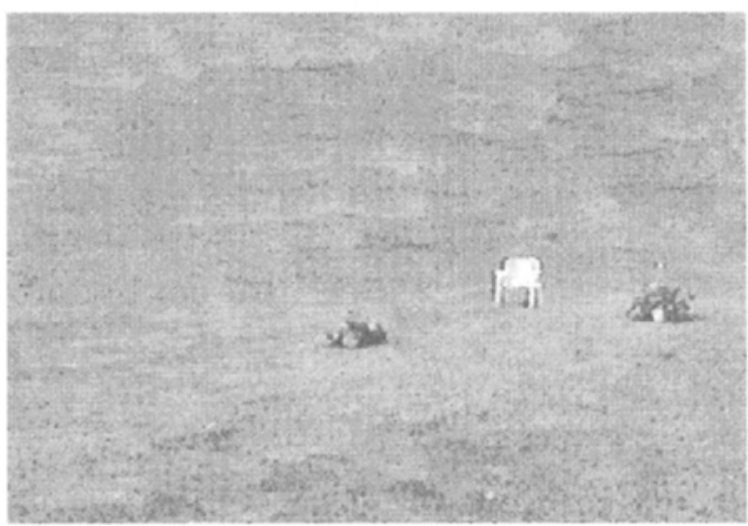

\section{Lm Shift}

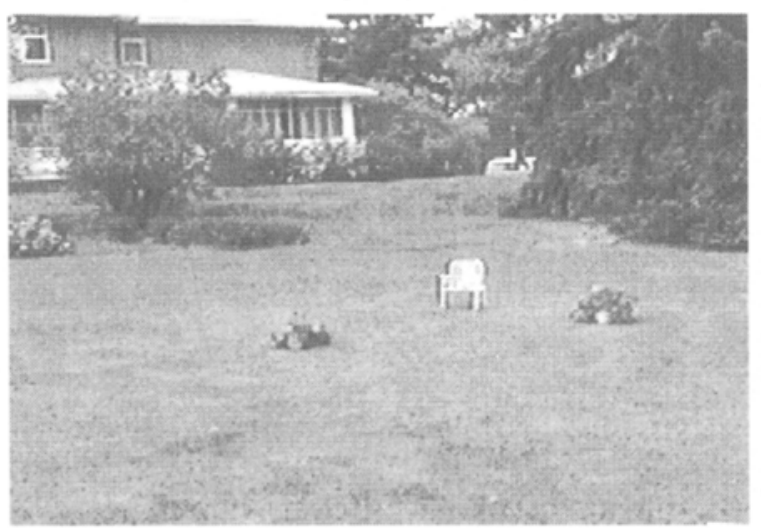

No Lms

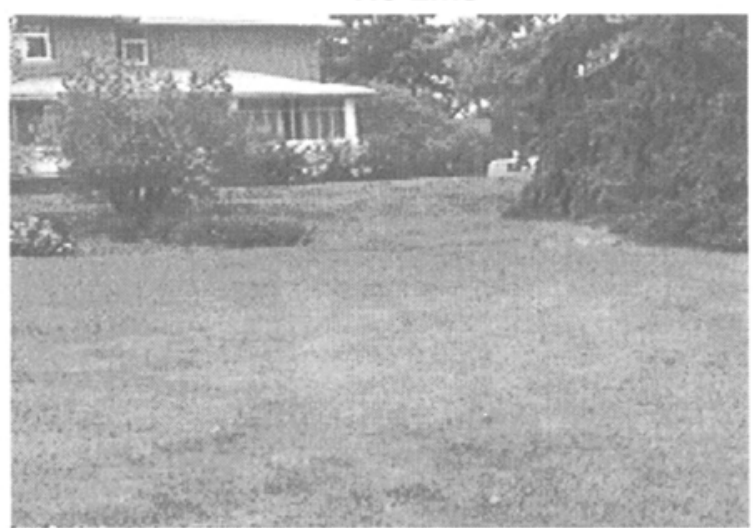

None

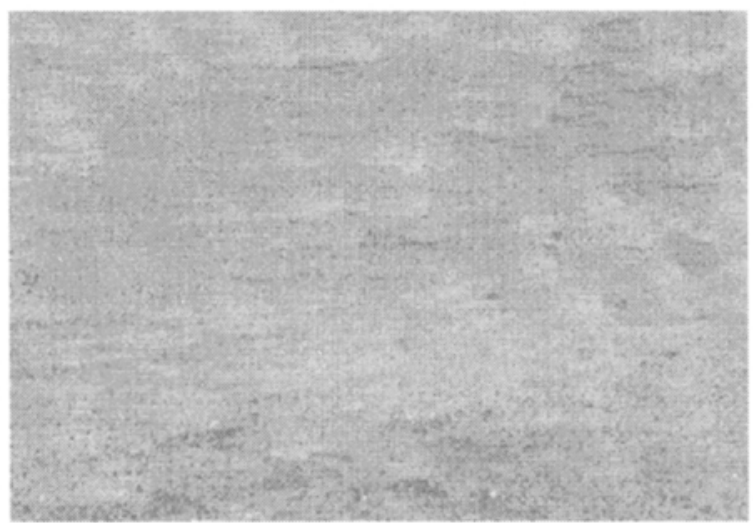

\section{Bk Swap}

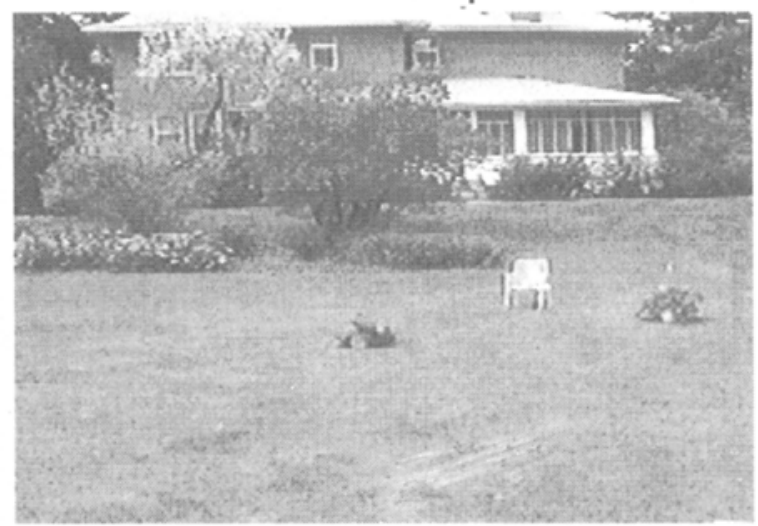

Figure 2. An example of control and test images used in Experiment $1 . \mathrm{Lm}=$ landmark; Bk $=$ background. The test images shown are based on Training View 4 . Tests based on other training views were also presented.

were removed, images in which all of the background information was removed, and images in which both the landmarks and the background were removed. The subjects who participated in a transfer test series were tested with each of the six transfer images shown in Figure 3. All subjects were debriefed following the session.

\section{Data Recording and Analysis}

All data presented are from unreinforced control and test trials. Each response was recorded in terms of touch-screen coordinates that ranged from 0 to $640 \mathrm{in}$ the horizontal dimension and 0 to 480 in the vertical dimension. Analysis of individual trial performance 


\section{Transfer Images}

1

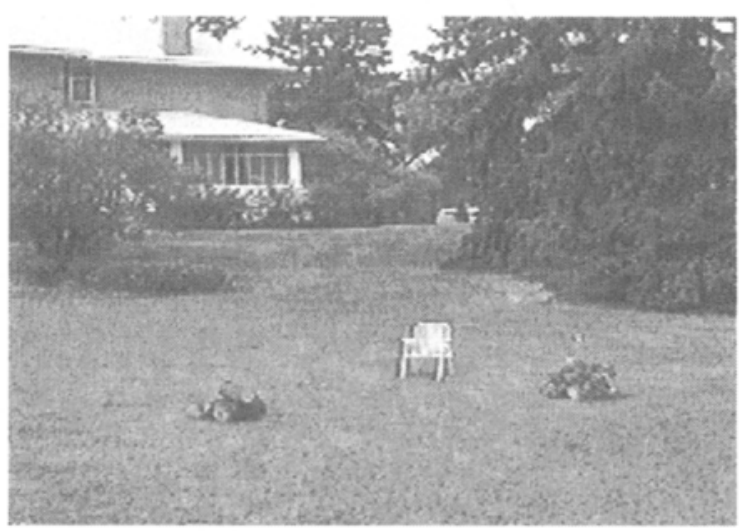

3

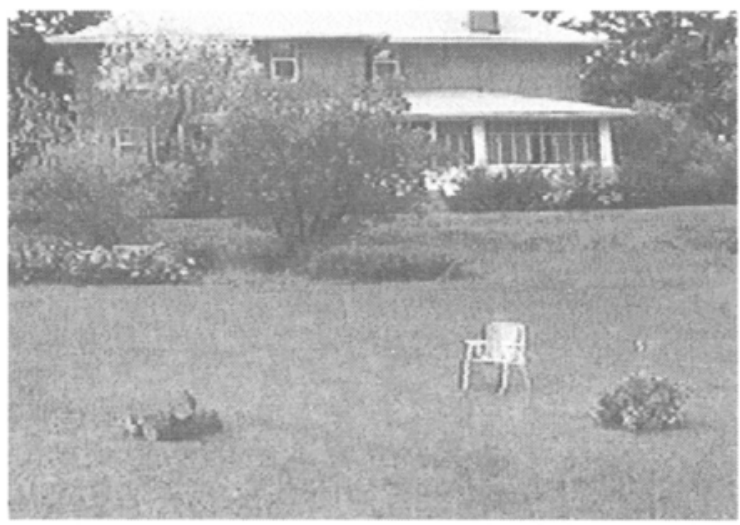

5

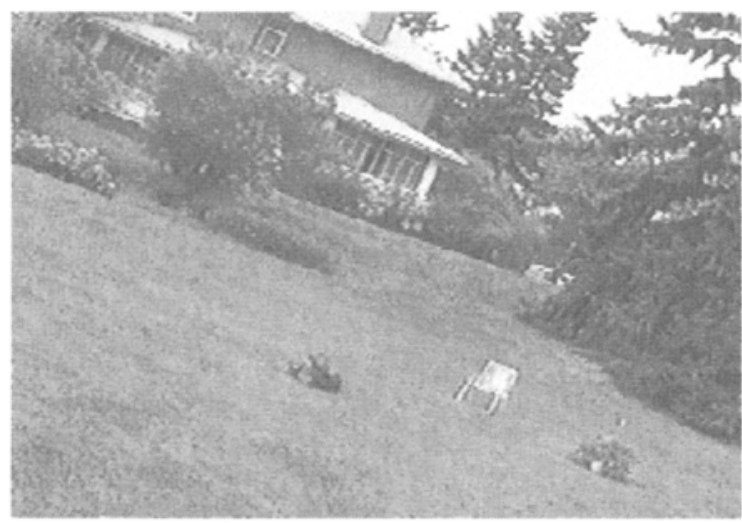

2

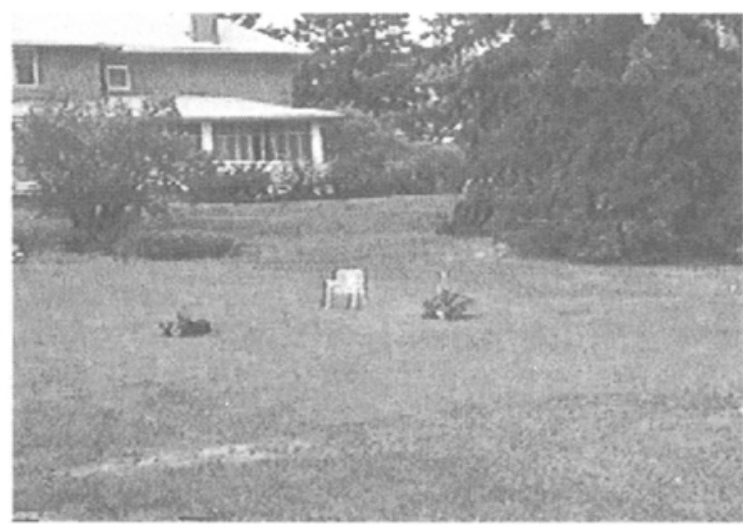

4

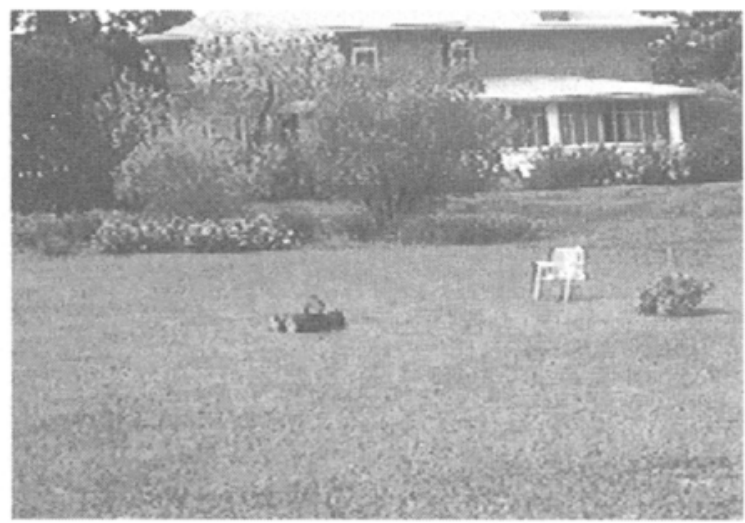

6

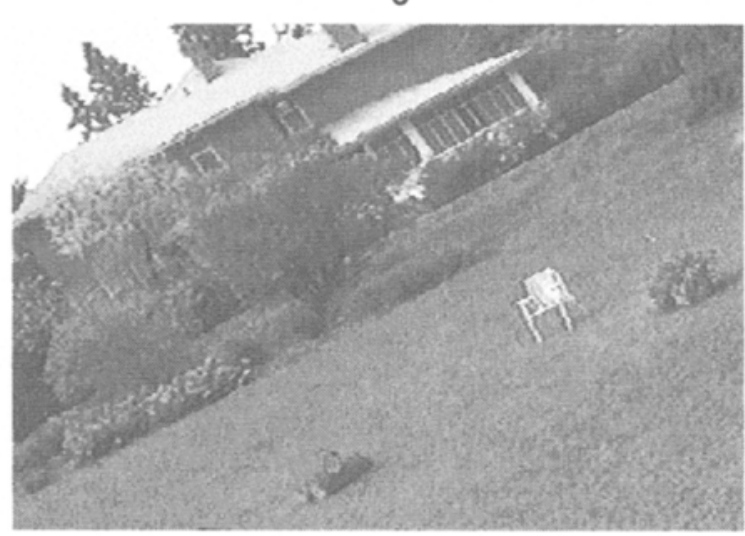

Figure 3. Images used during transfer testing in Experiment 1.

indicated that the pigeons often made a burst of peckıng ımmediately upon presentation of an image, and the location of the first few pecks was random with respect to the goal These pecks appeared to reflect orienting behavior rather than search behavior. In addition, casual observation indicated that the pigeons occasionally pecked directly at the frame of the touch screen or occasionally placed a foot on the bottom frame. These behaviors triggered the touch frame but did not appear to constitute search pecks. There- fore, the first three pecks made on every trial, and all pecks that fell within 20 pixels from the far edges of the search space, were excluded from the analysis of the data for pigeons.

Responses were considered correct if they were within 25 pixels of the center of the goal in both dimensions. For all tests conducted with images in which the visual information was manıpulated (e.g., landmarks or background removed or shifted), accuracy was determined according to the location of the goal in the image prior to these 
manipulations. Accuracy was calculated as correct responses divided by the total responses. All accuracy scores presented are averaged across all test trials and, except for the detailed results presented in the tables, are also averaged across views.

It should be noted that chance-level accuracy was very low. For example, if the subjects responded randomly on the screen, the probability of a response falling within the goal area was less than .01. For our determination of chance level, however, we took into account the possibility that the subjects might learn to respond only within the range of screen locations in which the goal was sometimes found. In this experiment, the goal locations used in training spanned a range of $230 \times 178$ pixels. If the subjects responded randomly in this range, the probability of a response falling in the $50 \times$ 50 pixel goal area was .06 .

For the shift tests and background swap tests, response coordinates were binned in the horizontal and vertical dimensions into 25 pixel units (approximately $1 \mathrm{~cm}$ ) that were relative to the center of the target area. Ten bins on either side of the target center were used for both dimensions. Any pecks farther than 250 pixels from the target center were placed in the 10th bin. Trials of the same type were combined across test sessions. These data were then used to compute the peak place of searching in the horizontal and vertical dimensions, using the iterated median procedure as described in Cheng (1989) and Spetch et al. (1992). This procedure determines the middle of the highest region in the distribution. First, the median of the response distribution was calculated. Then, the median calculation was iterated by calculating it over the region in which the previous median was centered. For example, if the first calculation over a range of $1-18$ units yielded a median of 3 , the next calculation would be over the range of Units $1-5$. This process was re- peated until two consecutive iterated medians differed by less than .05 of a unit. The last calculated median was taken to be the peak of the distribution.

For all statistical tests, our criterion for significance was $p<.05$. All a posteriori multiple comparisons used the Newman-Keuls test.

\section{Results}

\section{Removal of Individual Landmarks (Pigeons Only)}

Figure 4 shows the accuracy scores of each bird on tests in which individual landmarks were removed from the image as a proportion of their accuracy on control trials. The data are organized to show accuracy when a particular landmark was removed, together with accuracy when that landmark was the only local landmark present (i.e., the other two landmarks were removed). Strong control by a particular landmark would be indicated by low accuracy when the landmark was removed and high accuracy when it was presented alone. It is clear from the figure that the landmark exerting the most control varied across birds, with the dominant landmark being the flowers for Bird 66, the chair for Bird 20, and the logs for Birds 444 and 25.

\section{Removal of Landmarks, Background, or Both}

Figure 5 shows the mean accuracy of the pigeons (top panel) and the humans (bottom panel) on control trials

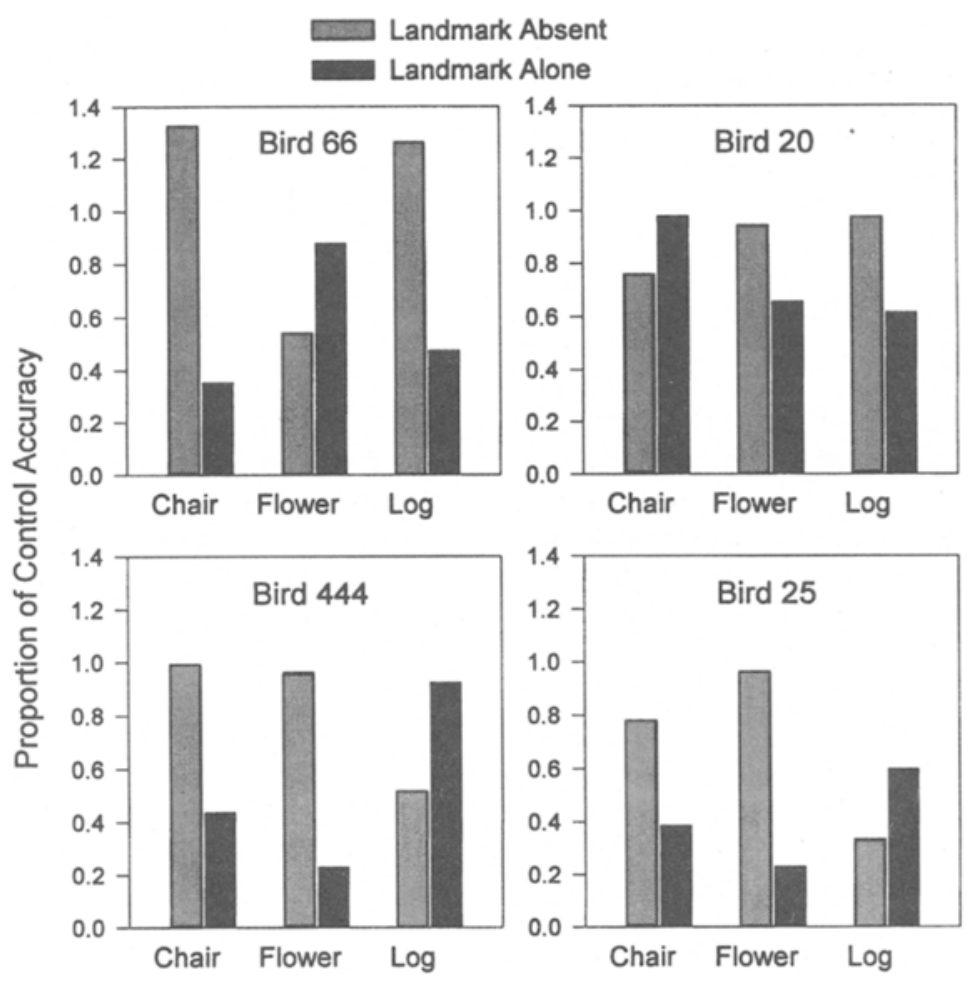

Figure 4. Accuracy as a proportion of control accuracy on tests with one of the proximal landmarks absent from the image or presented as the only proximal landmark in the image for each of the 4 birds in Experiment 1. 

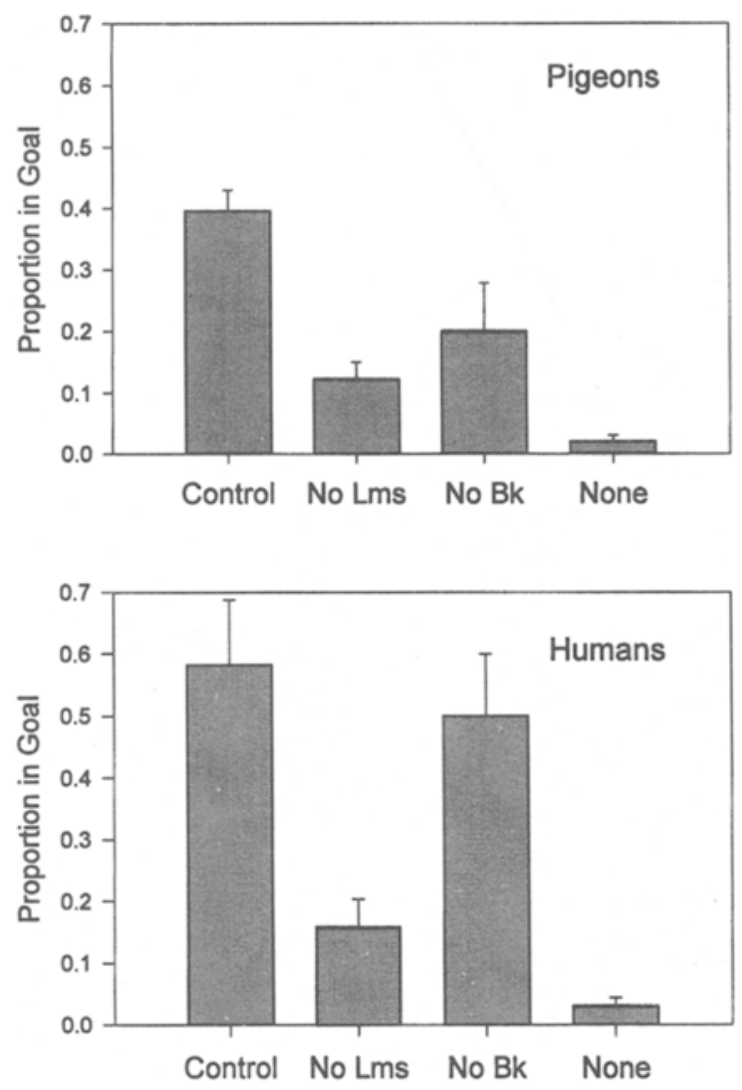

Figure 5. Mean proportion of responses in the goal on control trials, tests in which all proximal landmarks were removed (No Lms), tests in which all background cues were removed (No Bk), and tests in which both the proximal landmarks and the background were removed (None) for the pigeons and the humans in Experiment 1.

and on tests in which all three landmarks were removed (No Lms), the background was removed (No Bk), or both the landmarks and the background were removed (None).

For the pigeons, accuracy was disrupted by removal of either the background or the landmarks. An analysis of variance (ANOVA) revealed a significant effect of trial type $[F(3,9)=19.65]$, and multiple comparisons indicated that Control accuracy was significantly higher than accuracy on any of the three types of tests. In addition, accuracy on the No Bk tests was significantly higher than that on the None tests. Accuracy on No Lms tests was intermediate and was not significantly different from either the None tests or the No Bk tests.

For the human subjects, accuracy was significantly disrupted only by the removal of the landmarks. An ANOVA revealed a significant effect of trial type $[F(3,9)=19.89]$, and subsequent multiple comparisons revealed that accuracy on both Control and No Bk trials was higher than that on either No Lm or None trials. Accuracy did not differ significantly between Control and No Bk trials or between No Lm and None trials.

\section{Landmark Shifts (Pigeons Only)}

To assess whether the location of the pigeons' searching behavior shifted in the direction in which the landmarks were shifted, we calculated the horizontal and vertical peak places of searching on shift and control trials. The shift in searching was then assessed by calculating the difference between the control peak and the test peak in the shifted dimension and by assigning positive values if the difference was in the direction of the landmark shift and negative values if the difference was in the opposite direction. Averaged over the four views and the two dimensions, the average shift in searching was $0.57 \mathrm{~cm}$ in the direction of the shifted landmarks. This shift was significantly greater than $0[t(3)=4.63]$ but was significantly less than the average shift expected $(1.75 \mathrm{~cm})$ if the birds relied exclusively on the landmarks $[t(3)=9.64]$.

\section{Background Swap Tests (Pigeons Only)}

To assess whether the location of searching was influenced by visual cues in the background of the image, we compared peak place of searching on control trials for a given image with peak place of searching on test trials in which that image had its background swapped with another image. We then assessed whether a shift of the peak location in the appropriate direction occurred. For example, as can be seen in Figure 1, the goal location in Image 1 is to the left and below that in Image 4. Therefore, replacing the background of Image 4 with the background of Image 1 should result in a downward and leftward shift if the birds derive location information from the background. Shifts that were in the direction expected according to the background cues were assigned positive values, and those in the opposite direction were assigned negative values. Averaged across the two pairs of test images and across the horizontal and vertical dimensions, the subjects showed a mean shift of $0.56 \mathrm{~cm}$ in the expected direction. This shift was significantly greater than 0 $[t(3)=4.06]$ but significantly less than the average shift expected $(1.56 \mathrm{~cm})$ if the background was used exclusively to determine location $[t(3)=7.19]$.

\section{Transfer Tests With New Views}

Figure 6 shows the mean search accuracy of the pigeons (top panel) and the humans (bottom panel) on control trials with the six training views and on test trials with the six transfer views. The mean accuracy scores for each of the individual control and test views is shown in Table 1. The order of views in the table corresponds to the order shown in Figures 1 and 3.

For the pigeons, accuracy averaged across the six transfer views was significantly lower $(M=.143)$ than accuracy averaged across the six training views $(M=.458)[t(3)$ $=8.79]$. Although the overall accuracy on transfer tests was low, it was significantly higher than the .06 estimate of chance-level performance $[t(3)=3.78]$. Nevertheless, it is clear from Table 1 that the pigeons performed close to chance level with three of the six novel views. Moreover, the views with which they performed poorly in- 

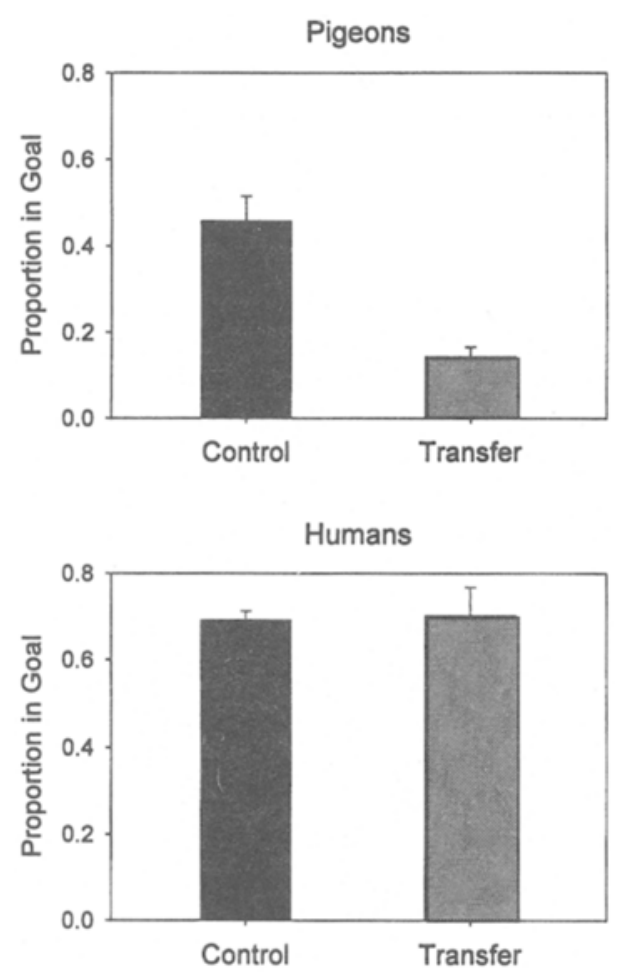

Figure 6. Mean proportion of responses in the goal on control tests (training views) and transfer tests (novel views) for the pigeons and the humans in Experiment 1.

cluded not only a rotated view (Image 6) but also two views (Images 1 and 2 ) that were in the same orientation as the training views. Thus, in general, the birds showed only weak evidence of being able to transfer the learned spatial information to a new view of the scene. By contrast, the human subjects showed no drop in accuracy when tested with the novel views. Accuracy averaged across the six training views $(M=.692)$ did not differ significantly from accuracy averaged across the six transfer views $(M=.702)[t(7)=0.23]$.

\section{Discussion}

Several interesting findings emerged regarding the way in which pigeons derive spatial information from images of a complex scene. First, the pigeons were able to learn the location of a goal in six images that presented different views of an outdoor scene. The goal location appeared to be encoded in terms of its relationship to both the proximal landmarks and the cues present in the background of the scene. This conclusion is based on the results of two kinds of tests. First, accuracy of searching was disrupted by removal of either the three proximal landmarks or the background. Second, tests in which the three landmarks were shifted or in which the background of one view was interchanged with the background of another view produced predictable shifts in the location of searching. However, in both cases, the magnitude of the shifts was less than expected if the manipulated cues were the only information used in deciding where to search. Thus, the pigeons appeared to use multiple sources of information when searching for the goal, a finding that is consistent with results from laboratory open-field studies of landmark-based search in pigeons (Cheng, 1992). The human subjects also showed a dramatic disruption of search accuracy when the three proximal landmarks were removed from the images; however, unlike the pigeons, they were not disrupted by removal of the visual stimuli in the background of the scene.

A second finding of interest was that the pigeons showed idiosyncratic preferences for one of the three proximal landmarks. Two of the birds showed strongest control by the logs, 1 by the chair, and 1 by the flowers. This finding is consistent with results from two previous studies using the touch-screen task. Spetch and Mondloch (1993) trained pigeons to find a goal that was roughly in the center of an array of four visually distinct geometric stimuli. Accurate searching in each pigeon was strongly controlled by only one or two of the landmarks, but the landmark exerting the most control varied across pigeons. Cheng and Spetch (1995) trained pigeons to find a goal that was close to both a small geometric landmark and one edge of a graphic frame. Tests indicated that some pigeons were controlled primarily by the landmark, some were controlled by both the landmark and frame, and 1 pigeon was controlled primarily by the frame. In both studies, the individual preferences appeared reliably across different types of tests. These idiosyncratic preferences are also reminiscent of the selective attention effects that have been reported when pigeons are trained with compound discriminative stimuli (e.g., Reynolds, 1961).

The finding that the pigeons in the present experiment did not show a uniform preference for the landmarks that were closer to the goal (logs or chair) over the landmark

Table 1

Screen Coordinates of the Target Location, Mean Proportions of Responses in the Goal, and Standard Errors of the Means for the Pigeons and the Humans for Each Control and Transfer Test Image in Experiment 1

\begin{tabular}{|c|c|c|c|c|c|c|}
\hline \multirow[b]{2}{*}{ Images } & \multicolumn{2}{|c|}{ Target Coordinates } & \multicolumn{2}{|c|}{ Pigeons } & \multicolumn{2}{|c|}{ Humans } \\
\hline & $x$ & $y$ & $M$ & $S E M$ & $M$ & $S E M$ \\
\hline \multicolumn{7}{|c|}{ Control } \\
\hline 1 & 331 & 351 & .350 & .059 & .719 & .086 \\
\hline 2 & 381 & 255 & .393 & .073 & .782 & .068 \\
\hline 3 & 365 & 383 & .593 & .106 & .601 & .100 \\
\hline 4 & 398 & 287 & .460 & .084 & .649 & .065 \\
\hline 5 & 218 & 319 & .452 & .051 & .780 & .070 \\
\hline 6 & 232 & 287 & .499 & .022 & .620 & .129 \\
\hline \multicolumn{7}{|c|}{ Transfer } \\
\hline 1 & 308 & 340 & .076 & .005 & .620 & .137 \\
\hline 2 & 232 & 279 & .069 & .024 & .637 & .031 \\
\hline 3 & 310 & 364 & .290 & .071 & .891 & .049 \\
\hline 4 & 381 & 270 & .226 & .085 & .621 & .157 \\
\hline 5 & 353 & 351 & .123 & .028 & .793 & .128 \\
\hline 6 & 398 & 335 & .075 & .016 & .652 & .081 \\
\hline
\end{tabular}

Note-Images are numbered in the order shown in Figures 1 and 3. Target coordinates are reported in pixels. 
that was farther from the goal (flowers) seems inconsistent with the results of a touch-screen study by Spetch and Wilkie (1994). In that study, pigeons were trained to find a goal in digitized images of a scene containing three landmarks that differed substantially in their distance to the goal. They found that pigeons uniformly preferred the landmark that was closest to the goal. Possible reasons why a uniform preference did not emerge in the present experiment will be discussed shortly.

The third interesting finding was that the pigeons showed a substantial drop in search accuracy when tested with novel views of the same scene. At best, they displayed only very marginal evidence of being able to transfer the learned spatial relationships to a new view of the scene. The human subjects, in contrast, showed complete transfer to the same new set of images. The results for the pigeons seem surprising, given that transfer between different views of an outdoor scene has been shown in other discrimination paradigms (Honig \& Stewart, 1988; Wilkie, Willson, \& Kardal, 1989). In those studies, pigeons were reinforced for responding to slides of one location and were not reinforced for responding to slides of another location. In the Honig and Stewart study, the $\mathrm{S}+$ and $\mathrm{S}-$ locations were different areas on the Dalhousie University campus that were each photographed from different viewpoints. The birds discriminated between the two locations and showed good transfer to novel slides of the two locations. In the Wilkie et al. (1989) study, the $\mathrm{S}+$ slides consisted of various views of a large geographic area in the vicinity of the psychology building on the University of British Columbia campus ("home" slides). The $\mathrm{S}$ - slides were similar views from various other geographic locations ("away" slides). The pigeons acquired the discrimination and transferred to novel views of home and away locations.

These studies and others (see Kendrick, 1992; Wilkie, Willson, \& MacDonald, 1992) suggest that pigeons are capable of forming a concept of a place presented in pictures and that pigeons can recognize that place in pictures that provide novel viewpoints. Why, then, would pigeons show such poor transfer to novel views of a scene in the present experiment? One possibility is that, in contrast to the place discrimination studies, transfer of accurate search behavior may not be possible unless the 3-D spatial relationship between the goal and the landmarks are extracted and applied to the new views. That is, transfer required not only that the pigeons recognize that the new views represented the same place as the trained views (as was assessed in the Honig \& Stewart, 1988, and Wilkie et al., 1989, studies) but also that they find the location of the goal in the new view of the scene. Moreover, since different views varied in both distance and direction, this would seem to require more than a simple transfer of the 2-D spatial relationships. The 2-D direction of search from landmarks needs to be adjusted for different viewing orientations, and the distance of search from landmarks needs to be adjusted according to the distance from which the scene is viewed. The pigeons in this experiment may not have responded to the $3-\mathrm{D}$ spatial relationships in the scene, and, hence, they would be unable to make the appropriate adjustments of distance and direction.

If the pigeons did not learn and transfer the 3-D spatial relationships between views, one might wonder how the pigeons were able to learn the task, given that different 2-D relationships were provided by each training view. One possibility is that the pigeons memorized the 2-D relationships between the visual stimuli and the goal in each of the six training views. Although this would seem to be a cumbersome way to acquire this task, pigeons have been shown to have excellent memories for visual details presented in pictures (e.g., Vaughan \& Greene, 1984), and, hence, they may be fully capable of doing so. In fact, their use of background cues as well as the more proximal landmarks may have reflected their need to discriminate among the views in order to respond to the correct 2 -D spatial relationships. The lack of any consistent preferences between the three proximal landmarks might also reflect attention to the 2-D relationships in each view rather than the 3-D relationship in the depicted scene. Although, in 3-D space, one landmark (flowers) was farther from the goal than were the other two landmarks, the difference in distance to the goal was not as consistently reflected in the 2-D spatial relationships provided by the six views.

\section{EXPERIMENT 2}

It is possible that the procedure and/or the stimuli used in Experiment 1 were not optimal for encouraging or allowing pigeons to extract the 3-D spatial relationships in images of a scene and apply these relationships to novel views. First, the set of training images was small enough so that memorization of the 2-D spatial relationships specific to each image would not be beyond the pigeons' ability. Moreover, studies of concept learning have found that pigeons often learn stimulus-specific rules when trained with a small set of exemplars, but they become more likely to learn a generalizable concept when trained with a larger set of exemplars (e.g., Wasserman \& Bhatt, 1992; Wright, Cook, Rivera, Sands, \& Delius, 1988). Experiment 2 therefore provided a much larger set of training views depicting a scene in an attempt to discourage memorization of view-specific relationships and to encourage the pigeons to solve the task on the basis of the underlying 3-D relationships. Second, extraction of the 3-D relationships in the views presumably requires that the birds perceive the depth information in the images, something that adult humans do readily. We do not know whether the pigeons perceived the depth in the images presented in Experiment 1. Some depth cues were ostensibly provided by the texture gradients and height of objects in the image, but recognition of the objects and knowledge of their relative sizes may be important for the perception of depth in these images. The pigeons used in Experiment 1 had never been outdoors and so would have little basis for recognizing any of the objects depicted in the scene. In Experiment 2, we sought to enhance the possibility that pigeons might perceive depth in the im- 
ages by using a scene that seemed to provide more perspective cues to depth. In addition, we used pigeons that had prior outdoor experience, which might provide a basis for recognizing some of the object categories (e.g., trees) depicted in the scene. Thus, the purpose of Experiment 2 was to find whether or not good transfer to novel views would occur under more optimal conditions.

\section{Method}

\section{Subjects}

Pigeons. Five female racing pigeons served as subjects. The p1geons had previous outdoor experience through their participation in races, but they were naive with respect to experımental procedures. They were maintained and housed as described in Experiment 1.

Humans. The human subjects were 11 undergraduate students ( 9 females, 2 males) ranging from 18 to 28 years of age. They participated in the experiment for credit in an introductory psychology class.

\section{Apparatus, Search Space, and Images}

For both the pigeons and the humans, the experimental apparatus and the search space were the same as those described for each species in Experiment 1. The images consisted of an outdoor scene taken from the Unıversity of Alberta campus during the winter. Trainıng images provided 28 differing views, which differed in distance to the goal as well as horizontal and vertical visual angle These 28 training images are shown in Figures 7 and 8 . In the vertical dimension, the scene was viewed from ground level or from the third and fourth stories of a building. The horizontal angle covered approximately $180^{\circ}$. For some views, the camera angle was rotated to $45^{\circ}$ or $315^{\circ}$ in either direction. The 28 trainıng views provided variation not only in viewing perspective but also in the absolute position of the goal on the touch screen.

The goal was located near a large black metal sculpture, approximately $12.5 \mathrm{~m}$ tall, that had two vertical components. The scene provided a complex assortment of additional cues, including the city's downtown skylıne, a windıng roadway, lampposts, trees, and buildings. A black box placed near the sculpture served as the goal marker, and it was present in the images only during preliminary training. The snow cover provided a reasonably uniform surface for the unmarked goal location.

The outdoor scene was videotaped, and images were generated and edited using the same procedures described in Experiment 1 . A large set of images was created, of which 28 were used for training and 18 for transfer testing. Determination of the touch-screen coordinates of the goal was accomplished using the procedure described in Experiment 1.

\section{Procedure for Pigeons}

Preliminary training. All pigeons were trained to eat from a raised ll luminated hopper and then were trained to peck at the monttor using the modified autoshaping procedure described in Experiment 1 . Initially, the images used to establish pecking consisted of a $2-\mathrm{cm}$ black square on a uniform light gray background (produced by cloning a patch of snow over an entire image). Once the pigeons reliably pecked at the black square, images of the scene with a $2-\mathrm{cm}$ black square centered over the goal marker were gradually introduced.

Search training. Durng the first stage of search trainıng, the black square marking the goal was decreased from $2.0 \mathrm{~cm}$ to $1.0 \mathrm{~cm}$ to $05 \mathrm{~cm}$ and then finally was eliminated, thereby requiring the bird to rely on landmark cues in the scene to locate the goal. Reductions and removal of the marker were accomplished by cloning surrounding snow over the marker Trainıng with the marker absent contınued until two criteria were met on each of three consecutive sessions (1) the bird completed at least 80 trials in each session, and (2) the proportion of pecks in the goal area was greater than .10 (estımated chance-level accuracy was only .03 , as described below).

During the second stage of search training, the peck requirement was increased to two pecks and then three pecks in the goal. Next, a requirement was added that the last two pecks recorded had to be consecutive pecks in the goal area.

In the final stage of training and for all subsequent baseline sessions, the percentage of reinforcement for successfully completed trials was decreased to $50 \%$. Each bird remained on $50 \%$ reinforcement for a minımum of 4 sessions and advanced to testing only after completing at least 80 trials on each of 3 consecutive sessions, and only if the proportion of pecks in the goal was at least .15 on each of these sessions. All other aspects of search trainıng were the same as in Experıment 1.

Tests. Each bird was given three test senes. Baseline sessions were interspersed between test sessions within and between series. The number of interspersed baseline sessions varied according to the bird's accuracy levels. The procedure used on control and test trials was the same as described in Experiment 1.

Series 1 and 3 tested for transfer to novel views. In Series 1, test sessions consisted of $50 \%$ reinforced trials with trainıng views, $25 \%$ unreinforced control trials with 12 of the traning views (the first 12 shown in Figure 7), and $25 \%$ unreinforced trials with test images that provided 12 new views of the scene (the first 12 mages shown in Figure 9). In Series 3, test sessions consisted of 50\% reinforced baseline trals with the training views, $25 \%$ unreinforced transfer tests with 6 novel views of the scene (the last 6 images shown in Figure 9), and $25 \%$ unreinforced tests with the same 6 transfer images but with all visual cues removed from the scene. These latter test images were created by cloning snow over the entire image (a sample of these is shown at the bottom of Figure 10). Series 2 tested for landmark control by the tall sculpture near the goal. Test sessions consisted of $50 \%$ reinforced trials with training views, $25 \%$ unreinforced control trials with 12 training views (the bottom two rows of images in Figure 7 and the top two rows of images in Figure 8 ), and $25 \%$ unreinforced trials composed from these same 12 trainıng views but with the sculpture shifted by $2 \mathrm{~cm}$ up, down, left, or right. Each type of shift was represented by three different views as shown in Figure 10. The extent of the shift in the represented 3-D space varied across views. In all series, each test image was presented for a total of 20 trials.

\section{Procedure for Humans}

The instructions and demonstration of the task were the same as described in Experiment 1, except that the images presented during the demonstration consisted of 2 of the training images from this experiment, 1 with the goal marker present and 1 with it absent. The subjects' program was identical to that used in Experiment 1, except for the tmages used and the sequence of trials. The first 12 trials presented 12 different trainıng images with the goal marker present. The next 12 trials presented the same 12 images but with the goal marker absent. The next 12 trials were randomly selected from the remaining set of 28 trainıng images with the goal marker present. For all remainıng baseline trials, the goal marker was absent. Trials 37.40 presented the 4 training images that had not yet been presented, and thereafter the 28 traming images were selected to occur equally often. Beginning on Trial 52, control and test trials were interspersed among baseline trials. Test trials presented the same set of 12 novel views used in Test Series 1 for the pigeons. Each test image was presented three tımes.

\section{Data Recording and Analysis}

Data were recorded and analyzed according to the procedures described in Experiment 1 However, estimated chance level was lower (.03), because a greater range of goal locations was provided 


\section{Training Images}
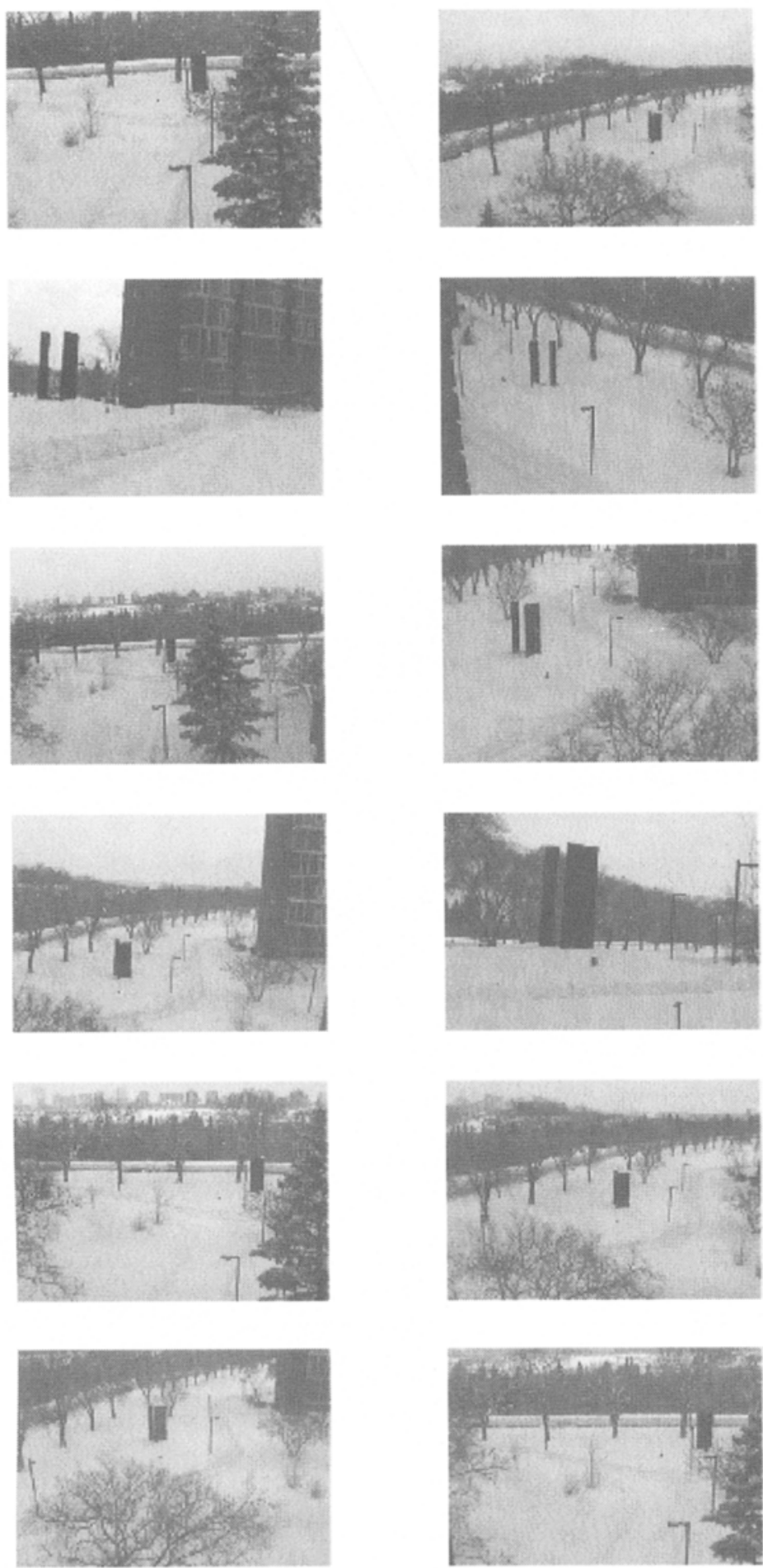
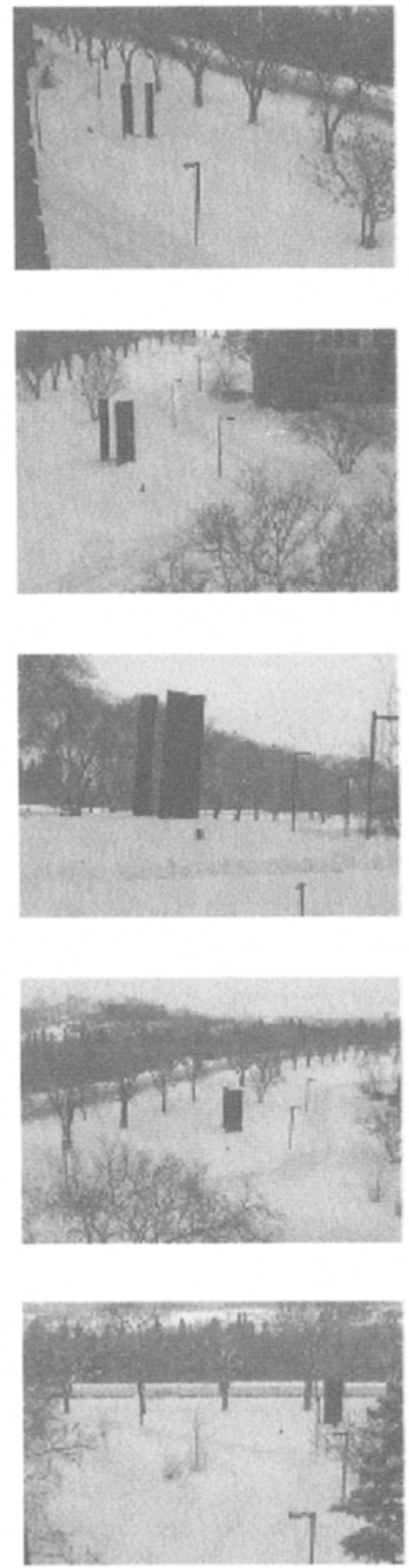
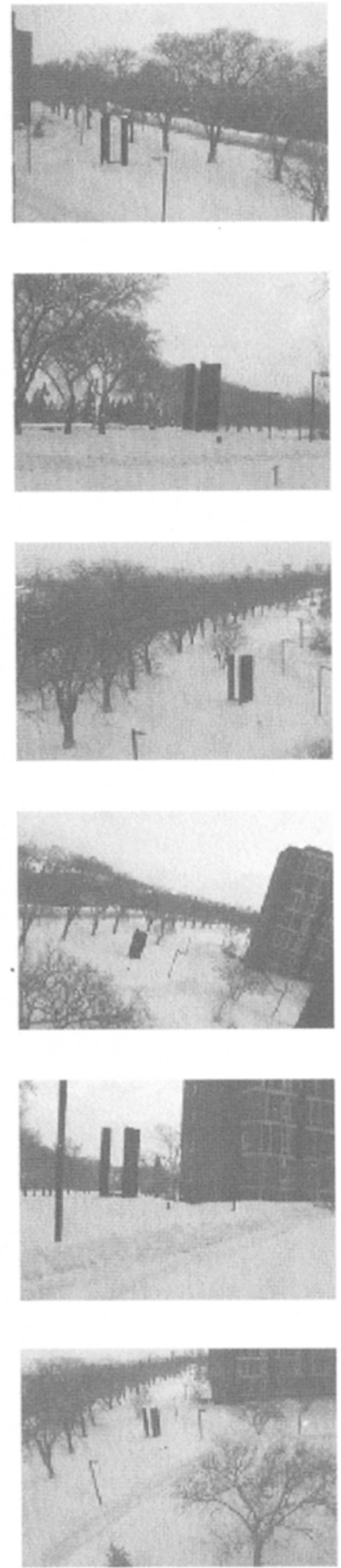

Figure 7. Images showing 18 of the 28 training views presented to the subjects in Experiment 2 . Images are numbered consecutively from left to right and then from top to bottom. The goal was located near the tall black sculpture. The small box used to mark the goal was edited out of the images after preliminary training and was not present during any baseline or test trials. 


\section{Training Images, Continued}
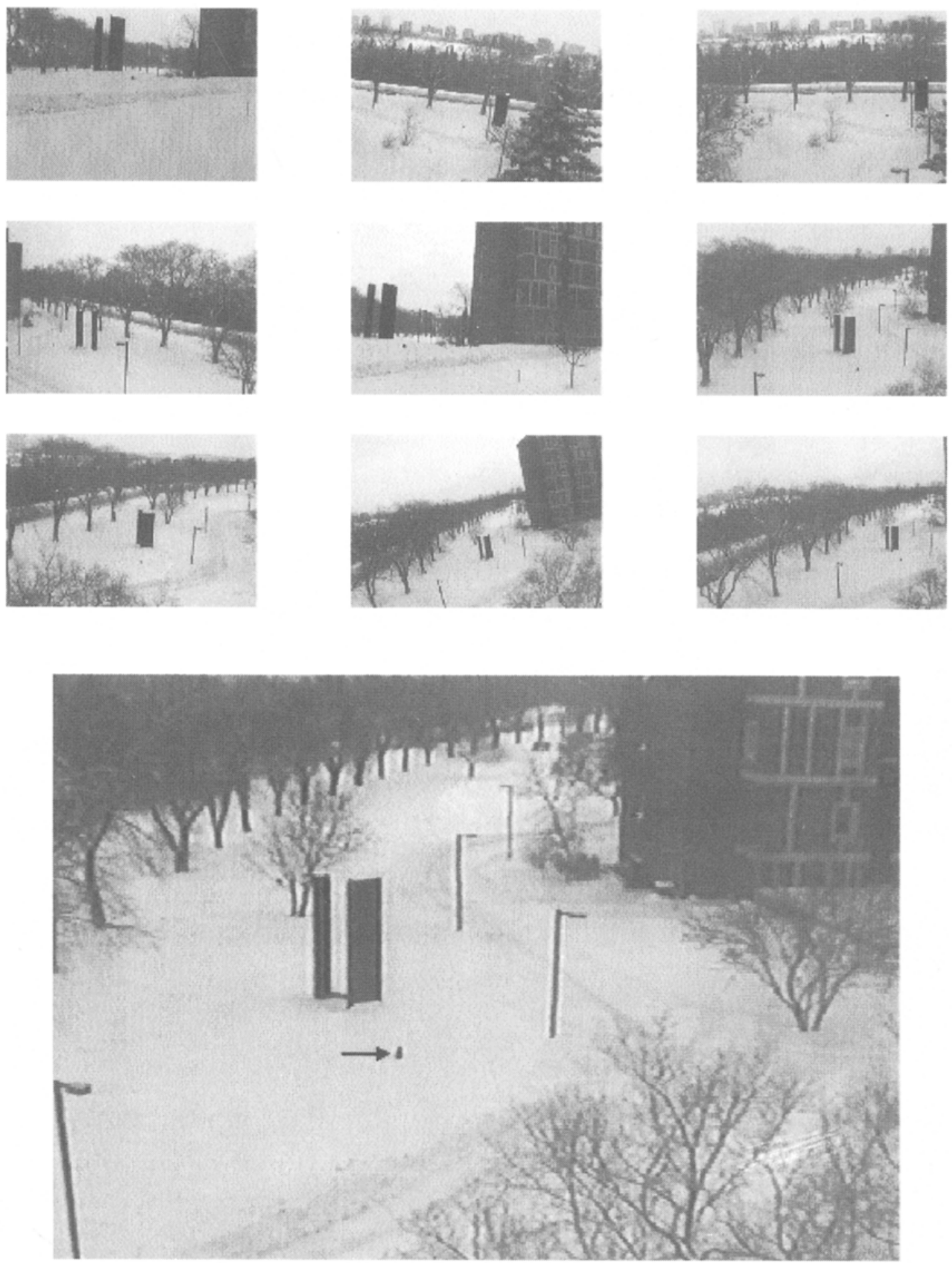

Figure 8. The 10 remaining training views in Experiment 2. The last image is shown in larger size so that details can be seen, and the goal is indicated with an arrow. The small box at the goal was edited out of the images following preliminary training. 


\section{Transfer Images}
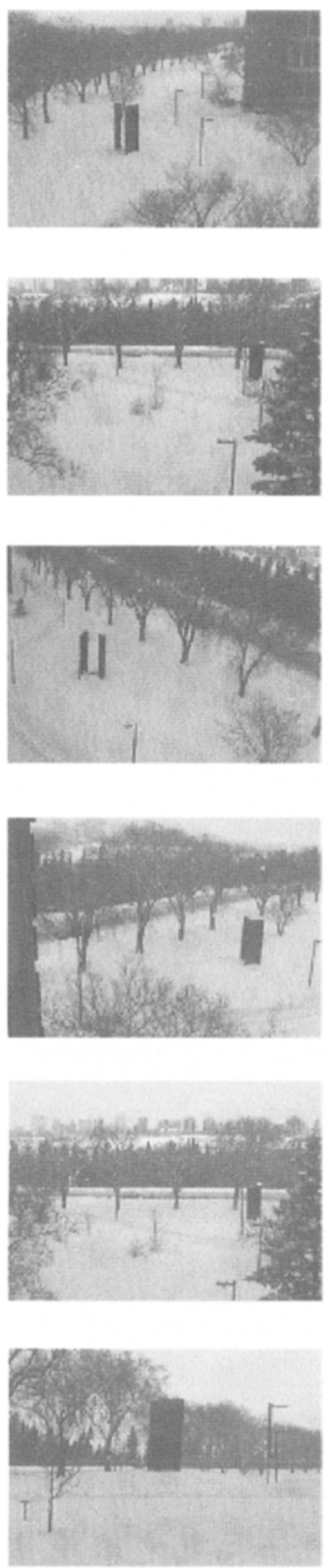
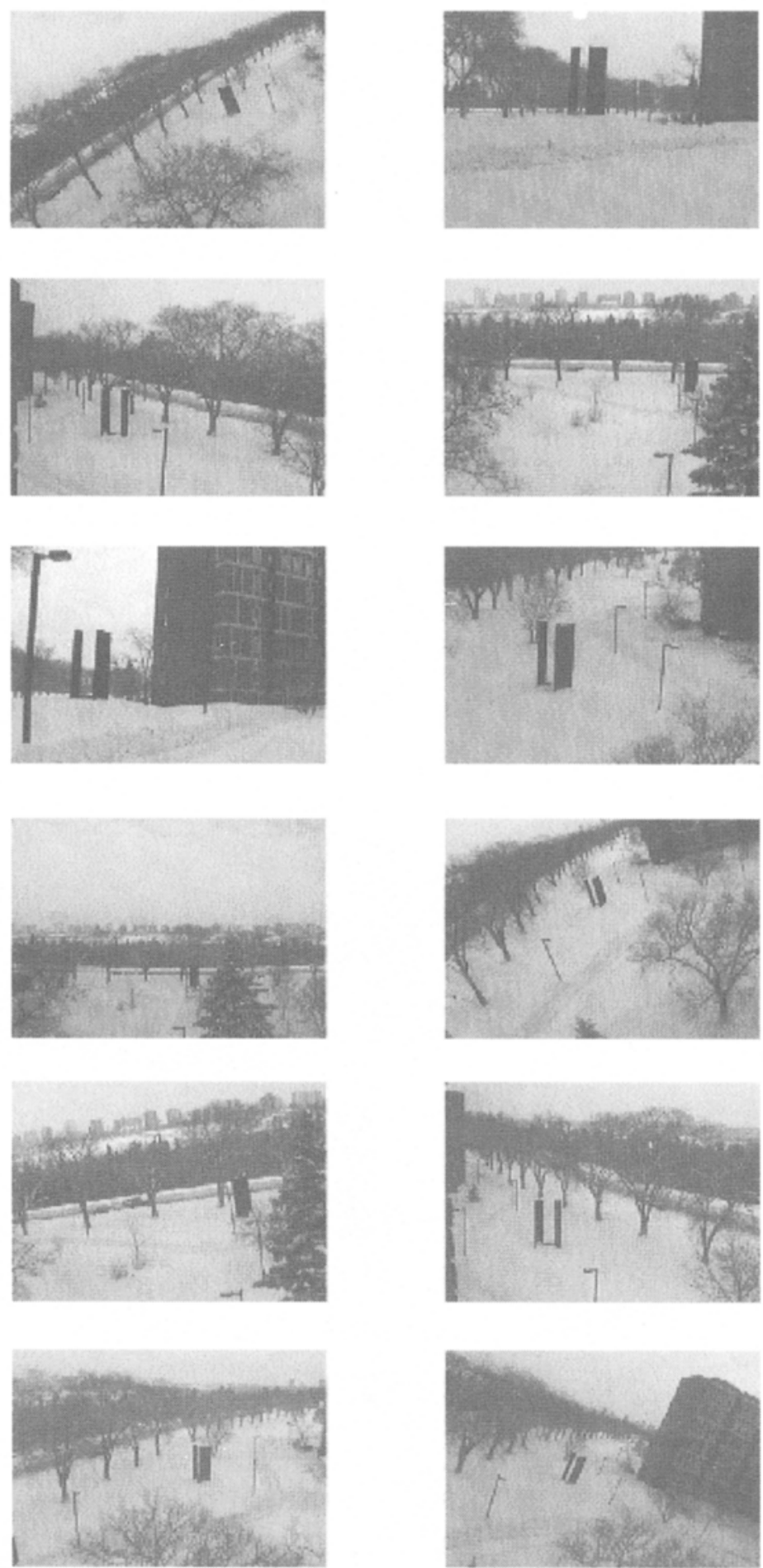

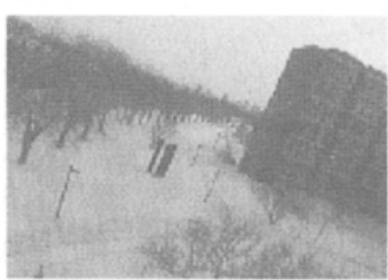

Figure 9. Novel views used for transfer testing in Experiment 2. The first 12 images were used for the humans and in Series 1 for the pigeons. The last 6 images were used in Series 3 for the pigeons. 


\section{Shift Tests}

\section{Left Shift}

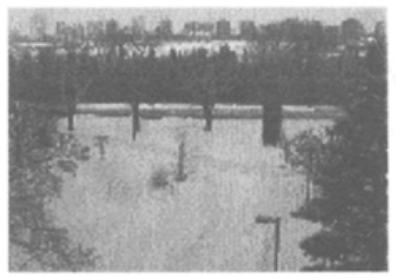

\section{Right Shift}

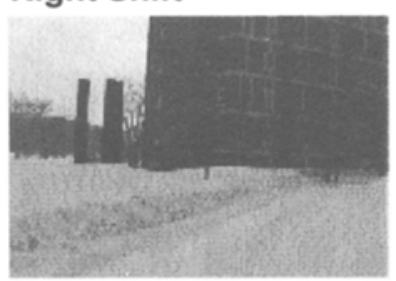

Upward Shift
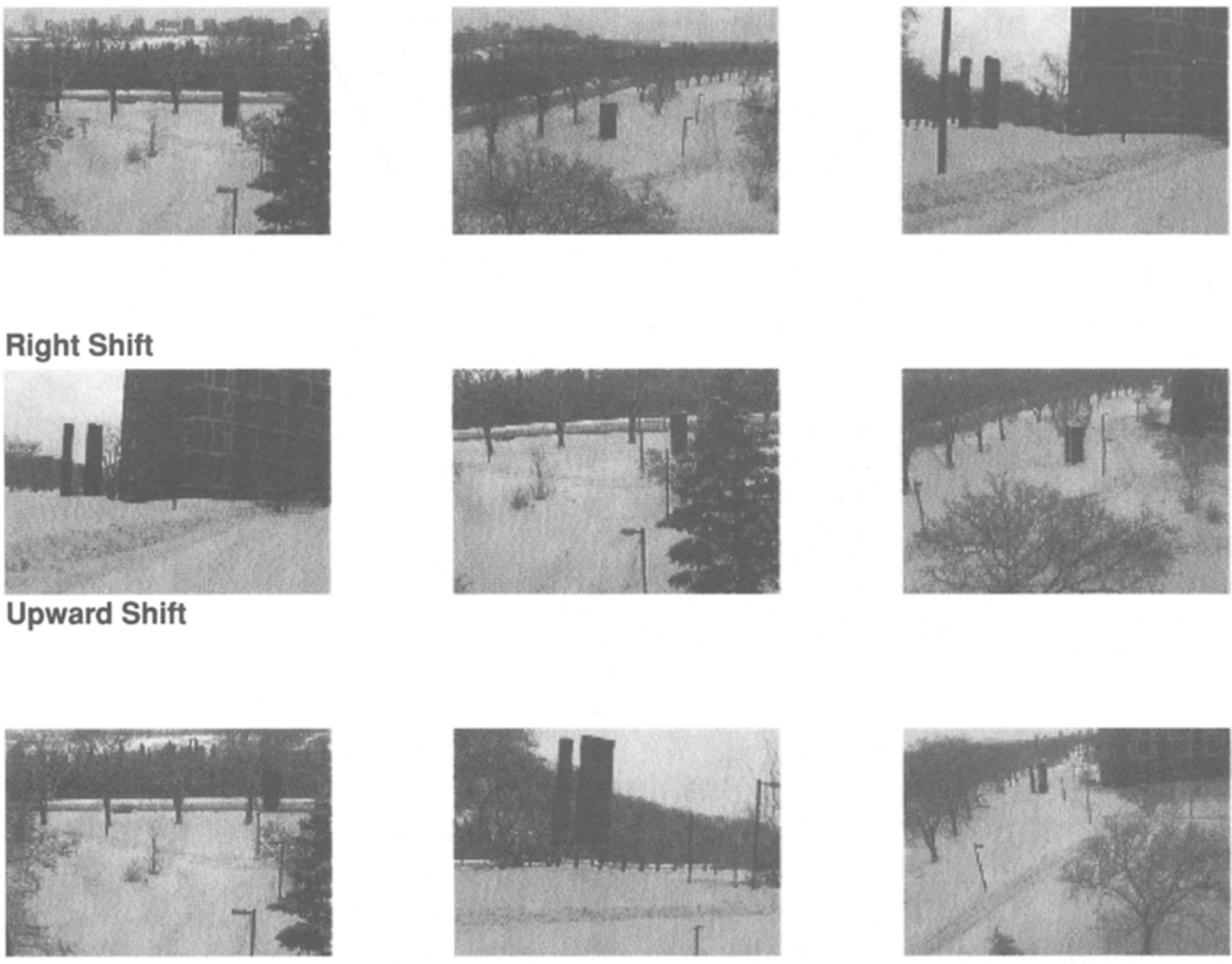

\section{Downward Shift}
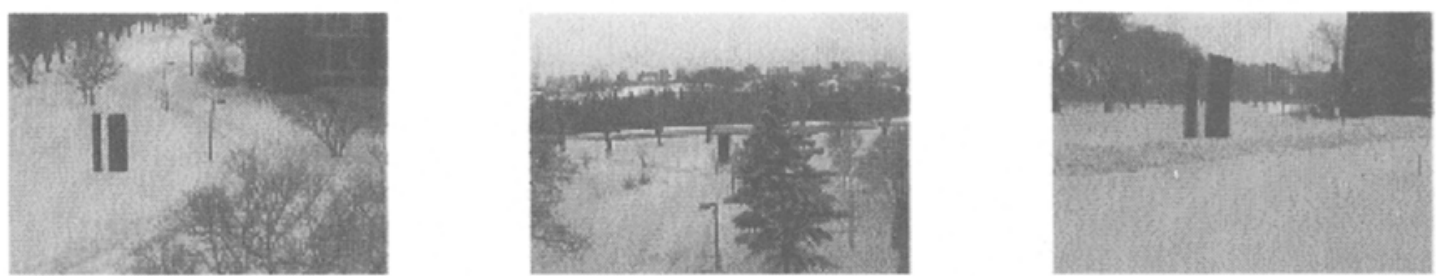

No Visual Cues
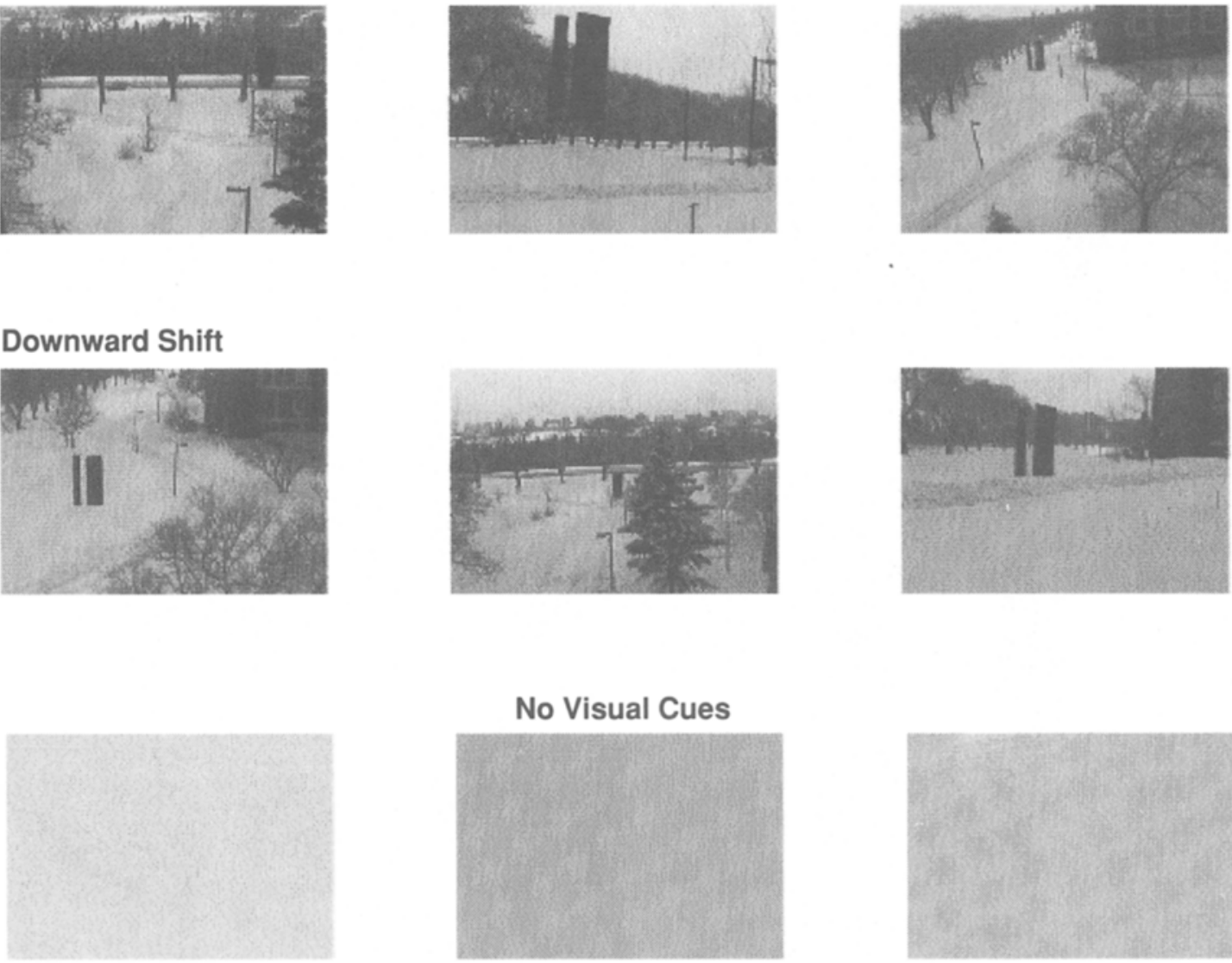
in the 28 training images used here than in the 6 training images used in Experiment 1.

\section{Results}

Acquisition of accurate searching by the pigeons was slow. Two of the pigeons failed to meet criterion within 200 sessions following the start of search training, and they were dropped from the study. The remaining 3 birds required 89,112 , and 114 sessions from the start of search training until they were ready for testing.

\section{Transfer to Novel Views}

Figure 11 shows the results of the transfer tests for the pigeons (top panel) and the humans (bottom panel). The left two bars for the pigeons show the results of Series 1 , which included tests with 12 training images (Control) and 12 novel images (Trans 1). The right two bars for the pigeons show the results of Series 3 , in which the pigeons were tested with 6 additional novel images (Trans 2) and with those same images with all visual information removed (No Visual). The human subjects did not receive the Series 3 tests. Accuracy scores for each individual control and test image are shown in Table 2.
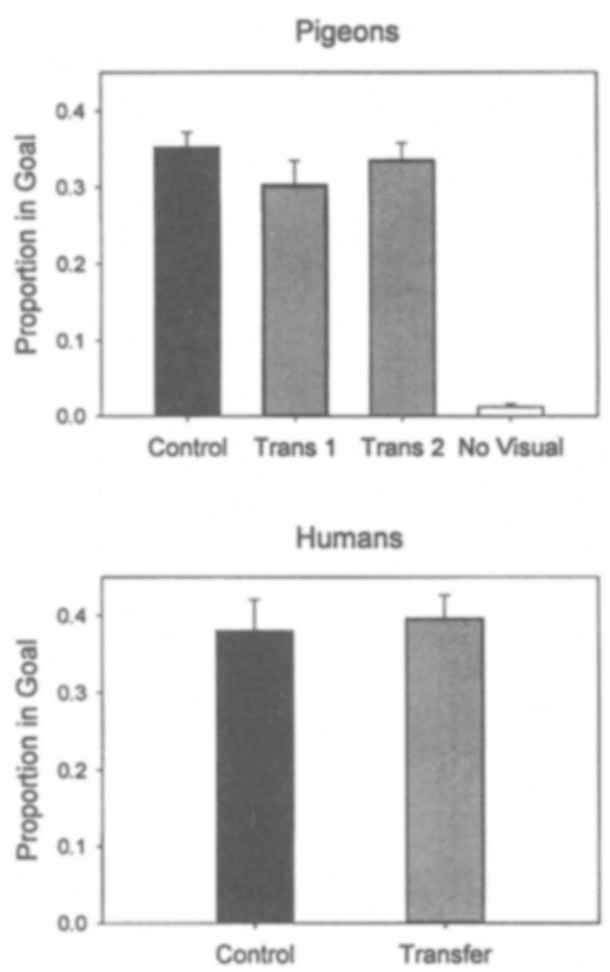

Figure 11. Transfer test results of Experiment 2 for the pigeons and the humans. For the pigeons, the left two bars show mean accuracy on control tests (training views) and transfer tests with the novel views presented during Series 1 (Trans 1). The right two bars show the pigeons' mean accuracy on the transfer tests with novel views (Trans 2 ) and the tests with images containing no visual cues (No Visual) during Series 3 . The human subjects received Series 1 tests only.
Table 2

Screen Coordinates of the Target Location for All Training and Transfer Images, Mean Proportions of Responses in the

Goal, and Standard Errors of the Means for the Pigeons and the Humans for Images Used for Control and Transfer Testing in Experiment 2

\begin{tabular}{|c|c|c|c|c|c|c|}
\hline \multirow[b]{2}{*}{ Images } & \multicolumn{2}{|c|}{ Target Coordinates } & \multicolumn{2}{|c|}{ Pigeons } & \multicolumn{2}{|c|}{ Humans } \\
\hline & $x$ & $y$ & $M$ & SEM & $M$ & $S E M$ \\
\hline \multicolumn{7}{|c|}{ Training } \\
\hline 1 & 305 & 198 & .389 & .044 & .422 & .074 \\
\hline 2 & 422 & 306 & .352 & .067 & .580 & .058 \\
\hline 3 & 108 & 328 & .301 & .024 & .414 & .058 \\
\hline 4 & 195 & 270 & .616 & .061 & .289 & .084 \\
\hline 5 & 117 & 218 & .347 & .024 & .416 & .069 \\
\hline 6 & 392 & 342 & .332 & .060 & .303 & .072 \\
\hline 7 & 265 & 252 & .424 & .039 & .571 & .067 \\
\hline 8 & 196 & 270 & .261 & .063 & .271 & .055 \\
\hline 9 & 473 & 372 & .426 & .085 & .296 & .079 \\
\hline 10 & 210 & 372 & .275 & .035 & .350 & .093 \\
\hline 11 & 295 & 312 & .221 & .046 & .311 & .081 \\
\hline 12 & 208 & 328 & .296 & .029 & .335 & .076 \\
\hline 13 & 2 & 9 & 9 & .058 & & \\
\hline
\end{tabular}

.058

$.285 \quad .020$

$.252 \quad .051$

$.287 \quad .047$

$.283 \quad .013$

$.353 \quad .024$

$.484 \quad .064$

$280 \quad 303$

$479 \quad 278$

$112 \quad 334$

$125 \quad 325$

$390 \quad 370$

$336 \quad 330$

$358 \quad 340$

$509 \quad 320$

$255 \quad 290$

28

\begin{tabular}{lllllll} 
& \multicolumn{5}{c}{ Transfer } & \\
1 & 269 & 339 & .290 & .088 & .342 & .073 \\
2 & 450 & 252 & .339 & .027 & .448 & .097 \\
3 & 345 & 240 & .232 & .008 & .337 & .054 \\
4 & 420 & 214 & .402 & .062 & .311 & .068 \\
5 & 122 & 320 & .446 & .026 & .378 & .088 \\
6 & 415 & 241 & .477 & .048 & .468 & .057 \\
7 & 088 & 248 & .213 & .041 & .225 & .048 \\
8 & 252 & 342 & .168 & .051 & .303 & .087 \\
9 & 260 & 351 & .175 & .052 & .182 & .056 \\
10 & 468 & 340 & .150 & .042 & .494 & .082 \\
11 & 325 & 359 & .202 & .055 & .764 & .057 \\
12 & 330 & 198 & .547 & .128 & .499 & .058 \\
13 & 413 & 291 & .332 & .027 & & \\
14 & 380 & 306 & .352 & .028 & & \\
15 & 122 & 322 & .447 & .063 & & \\
16 & 292 & 265 & .113 & .041 & & \\
17 & 374 & 306 & .399 & .026 & & \\
18 & 254 & 304 & .370 & .071 & &
\end{tabular}

Note-Images are numbered in the order shown in Figures 7, 8, and 9. Target coordinates are reported in pixels.

For the pigeons during Series 1, overall mean accuracy on transfer tests with novel views (.303) was only slightly lower than the overall mean accuracy on control tests (.353), and the difference failed to reach significance $[t(2)=3.84]$. Moreover, accuracy on transfer tests was significantly higher than expected by chance $(.03)[t(2)=$ 8.58]. During Series 3, the overall mean accuracy on 
novel view tests (.336) was again significantly higher than expected by chance $[t(2)=13.73]$ and was also higher than the overall mean accuracy on tests with the visual information removed from the images $(.012)[t(2)=$ 12.59]. Accuracy with no visual cues was significantly lower than the estimated chance level $[t(2)=4.99]$.

The human subjects also showed good transfer to novel views of the scene. Their overall mean accuracy on control trials (.380) did not differ significantly from their overall mean accuracy on transfer test trials (.401) $[t(10)=0.89]$.

\section{Landmark Shifts (Pigeons Only)}

Shifts of the large landmark near the goal (i.e., the sculpture) produced small but significant shifts in searching in the direction of the landmark shift. Horizontal shifts of the landmark by $2 \mathrm{~cm}$ resulted in significant shifts of searching in the appropriate direction $(M=0.67 \mathrm{~cm})$ $[t(2)=8.64]$, whereas $2-\mathrm{cm}$ vertical shifts of the landmark did not produce significant shifts in the appropriate direction $(M=0.13 \mathrm{~cm})[t(2)=0.76]$. Closer inspection of the vertical shift results indicate that when the landmark was shifted down, the birds showed a significant downward shift in searching $(M=0.97)[t(2)=$ 5.30]. However, when the landmark was shifted up, the birds showed a nonsignificant shift in the wrong direction $(M=0.70 \mathrm{~cm}$ down $)[t(2)=1.37]$. Averaged across all directions of landmark shifts, $2-\mathrm{cm}$ shifts of the landmark resulted in $0.40-\mathrm{cm}$ shifts in searching in the appropriate direction, which was significantly greater than $0[t(2)=8.20]$.

\section{Discussion}

These results indicate that, at least under some conditions, pigeons can transfer accurate search behavior to novel views of a scene. On unreinforced tests with novel views, the pigeons searched with accuracy levels comparable to their accuracy levels with the trained views. Their accuracy levels with the novel views were also well above the estimated chance levels and the level of accuracy they achieved when all visual cues were removed from the images. The human subjects again showed complete transfer to the novel views, searching as accurately with the novel views as with the training views. Interestingly, both the pigeons and the humans showed considerable variability in accuracy across views both in the training and transfer sets, suggesting that some perspectives were more difficult than others.

The shift tests revealed that the pigeons used the tall sculpture near the goal as a landmark, but they used other cues as well. The birds shifted only partway with the sculpture when it was shifted left, right, or down. The birds did not follow the sculpture when it was shifted upward. Although inspection of Figure 10 suggests that two of the upward-shift images might be problematic because the landmark appears to "float" above the snow, the birds also failed to follow the upward landmark shift even in the image in which the landmark remained in the snow. At present, we have no explanation for the anomalous results of the upward landmark shifts.

\section{GENERAL DISCUSSION}

In these experiments, the pigeons learned to locate a goal in images of an outdoor scene viewed from varying perspectives. The scenes used in each experiment provided a rich set of visual cues that maintained a constant relationship to the goal in the represented 3-D space. The 2-D relationship of these cues to the goal, however, varied across images. The pigeons in both experiments learned the task, and the cue manipulation tests conducted in Experiment 1 indicated that they used both landmarks near the goal and additional background cues in searching for the goal. The results of the cue-manipulation test conducted in Experiment 2 also suggest that search behavior was controlled by more than one source of spatial information, because search shifted only partway toward the shifted landmark. This evidence that search behavior is controlled by multiple sources of spatial information is consistent with results from search tasks conducted on the laboratory floor (Cheng, 1992).

Although the pigeons in both experiments learned to search accurately in images depicting varying views of a scene, only in Experiment 2 did the pigeons show strong transfer of accurate search to novel views of the same scene. The weak transfer in Experiment 1 suggests that the pigeons may have memorized the 2-D spatial relationships between landmark cues and the goal specific to each of the six training views, rather than learning the 3-D spatial relationships in the represented scene. On the other hand, the occurrence of strong positive transfer in Experiment 2 suggests that, under some circumstances, pigeons may be able to learn the 3-D spatial relationship depicted in different training views of a scene.

Although the positive transfer to novel views seen in Experiment 2 suggests the use of 3-D information, we cannot conclusively rule out the use of a 2-D strategy. Each transfer view was novel and differed to some extent from any of the training views. However, the training set used in Experiment 2 was large, and some transfer views looked quite similar to one or more of the training views, differing only by a lateral or vertical displacement. In such cases, transfer could be obtained if the pigeons (1) memorized the 2-D relationships in each training image or in subsets of training images, (2) generalized from the most similar training image to the transfer image, and (3) shifted their searching in accordance with the horizontal and/or vertical displacement of the visual information from the training to the transfer image (or, in other words, searched at the same 2-D vector from the landmark[s] as in the training image). Such a strategy could account for accurate performance with several of the transfer images, but it does not appear to account well for all of the transfer results. For example, Transfer Image 2 appears most similar to Training Image 2, but the orientation is rotated, so that vectors from landmarks to the goal differ (unless one 
uses information in the picture to adjust the directional frame of reference). Yet accuracy with Transfer Image 2 was not substantially lower than accuracy with Training Image 2. Transfer Image 18 appears most similar to Training Image 12 , but it is viewed from a different vantage point (as can be seen by the position of the tree in the bottom of the image and by the appearance vs. occlusion of the second vertical component of the sculpture). Generalization between these images and application of the same vector from any of the salient visual cues (e.g., the building, the tree at the bottom, or the sculpture) should produce systematic errors in search location. Yet accuracy was slightly higher with the transfer image than with the trained image. Thus, at least some of the transfer results seem more consistent with the possibility that the birds did in fact attend to the 3-D relationships in the scene. Nevertheless, unequivocal evidence of 3 -D processing is difficult to provide, and it likely will require the convergence of results from many different experiments.

Several differences between the two experiments could underlie the difference in transfer results. First, the birds in Experiment 2 were trained with many more different views of the scene than were the birds in Experiment 1 (28 vs. 6), which may have discouraged memorization of the spatial relationships specific to each view and facilitated attention to the 3-D relationships that were constant across views. Training with more exemplars also enhances stimulus generalization (e.g., Wasserman et al., 1996). Second, to the human eye at least, the scene depicted in Experiment 2 seems to provide more depth cues. Third, the pigeons in Experiment 2, unlike those in Experiment 1 , had prior outdoor experience and therefore had some basis for recognizing at least some types of objects depicted in the scene. Determining which of these factors is responsible for the difference in results is a challenge for future research.

\section{REFERENCES}

BroDBEck, D. R. (1994). Memory for spatial and local cues. A comparison of a storing and a nonstoring species. Animal Learning \& Behavior, 22, 119-133.

ChenG, K. (1988). Some psychophysics of the pigeon's use of landmarks. Journal of Comparative Physiology A, 162, 815-826.

CHeng, K. (1989). The vector sum model of landmark use. Journal of Experimental Psychology Animal Behavior Processes, 15, 366-375.

CHENG, K. (1992). Three psychophysical principles in the processing of spatial and temporal information. In W. K. Honig \& J. G. Fetterman (Eds.), Cognitive aspects of stimulus control (pp. 69-88). Hıllsdale, NJ. Erlbaum.

Cheng, K. (1994). The determination of direction in landmark-based spatial search in pigeons: A further test of the vector sum model. $\mathrm{An}$ imal Learning \& Behavior, 22, 291-301.

CHENG, K., \& SHERRY, D. (1992). Landmark-based spatial memory in birds: The use of edges and distances to represent spatial positions. Journal of Comparative Psychology, 106, 331-341.
Cheng, K., \& Spetch, M. L. (1995). Stimulus control in the use of landmarks by pigeons in a touch-screen task. Journal of the Experimental Analysis of Behavior, 63, 187-201.

Collett, T. S. (1992). Landmark learning and guidance in insects. Philosophical Transactions of the Royal Society of London. Series B, 337, 295-303.

GALLISTEL, C. R. (1990). The organization of learning. Cambridge, MA: MIT Press.

Honig, W. K., \& Stewart, K. E. (1988). Pigeons can discriminate locations presented in pictures. Journal of the Experimental Analysis of Behavior, 50, 541-551.

Kendrick, D. F. (1992). Pigeon's concept of experienced and nonexperienced real-world locations: Discrimination and generalization across seasonal varıation. In W. K. Honig \& J. G. Fetterman (Eds.), Cognitive aspects of stimulus control (pp 113-134). Hillsdale, NJ: Erlbaum.

REYNOLDS, G. S. (1961). Attention in the pigeon. Journal of the Experimental Analysis of Behavior, 4, 203-208.

SPETCH, M L. (1995). Overshadowing in landmark learning: Touchscreen studies with pigeons and humans. Journal of Experimental Psychology: Animal Behavior Processes, 21, 166-181.

SPetch, M. L., Cheng, K., \& MacDonald, S. E. (1996). Learning the configuration of a landmark array: I. Touch-screen studies with plgeons and humans. Journal of Comparative Psychology, 110, 55-68.

Spetch, M. L., Cheng, K., MacDonald, S. E., Linkenhoker, B. A., KELLY, D. M., \& DOERKSON, S R. (1997). Use of landmark configuration in pigeons and humans: II. Generality across search tasks. Journal of Comparative Psychology, 111, 14-24.

SPETCH, M. L., Cheng, K., \& Mondloch, M. V. (1992). Landmark use by pigeons in a touch-screen spatial search task. Animal Learning \& Behavior, 20, 281-292.

SPetch, M. L., \& Edwards, C. A. (1988). Pigeons', Columba livia, use of global and local cues for spatial memory. Animal Behaviour, 36, 293-296.

SPETCH, M. L., \& Mondloch, M. V. (1993). Control of pigeons' spatial search by graphic landmarks in a touch-screen task. Journal of Experimental Psychology' Animal Behavior Processes, 19, 353-372.

SPETCH, M. L., \& Wilkie, D M. (1994). Pigeons' use of landmarks presented in digitized images. Learning \& Motivation, 25, 245-275

VAughan, W., JR., \& Greene, S. L. (1984). Pigeon visual memory capacity. Journal of Experimental Psychology Animal Behavior Processes, 10, 256-271.

Wasserman, E. A., \& Bhatt, R. S. (1992) Conceptualization of natural and artificial stımuli by pıgeons. In W. K. Honig \& J. G. Fetterman (Eds.), Cognitive aspects of stimulus control (pp. 203-223). Hillsdale, NJ: Erlbaum.

Wasserman, E. A., Gagliardi, J. L., Cook, B. R., Kirkpatrick Steger, K., Astley, S. L., \& Biederman, I. (1996). The pigeon's recognition of drawings of depth-rotated stimuli. Journal of Experimental Psychology Animal Behavior Processes, 22, 205-221

Wilkie, D. M., MAK, T., \& SAKsida, L. M. (1994). Pigeons' landmark use as revealed in a "feature positive," digitized landscape, touchscreen paradigm. Behavioural Processes, 32, 39-56.

Wilkie, D. M., Willson, R. J., \& Kardai,, S. (1989). Pigeons discrıminate pictures of a geographic location. Animal Learning \& Behavior, 17, 163-171.

Wilkie, D. M., Willson, R. J., \& MacDonald, S. E. (1992). Animals' perception and memory for places. In W. K. Honı \& J. G. Fetterman (Eds.), Cognitive aspects of stimulus control (pp. 89-112). Hillsdale, $\mathrm{NJ}$ : Erlbaum.

Wright, A. A., COOK, R. G., Rivera, J. J., SAnds, S. F., \& Delius, J D. (1988). Concept learning by pigeons: Matching-to-sample with tria!unique video picture stımulı Animal Learning \& Behavior, 16, 436-444. 